\title{
Can ionic concentration changes due to mechanical deformation be responsible for the neurostimulation caused by focused ultrasound? a simulation study
}

To cite this article before publication: Vladimir Filkin et al 2021 Physiol. Meas. in press https://doi.org/10.1088/1361-6579/ac2790

\section{Manuscript version: Accepted Manuscript}

Accepted Manuscript is "the version of the article accepted for publication including all changes made as a result of the peer review process, and which may also include the addition to the article by IOP Publishing of a header, an article ID, a cover sheet and/or an 'Accepted Manuscript' watermark, but excluding any other editing, typesetting or other changes made by IOP Publishing and/or its licensors"

This Accepted Manuscript is @ 2021 Institute of Physics and Engineering in Medicine.

As the Version of Record of this article is going to be / has been published on a gold open access basis under a CC BY 3.0 licence, this Accepted Manuscript is available for reuse under a CC BY 3.0 licence immediately.

Everyone is permitted to use all or part of the original content in this article, provided that they adhere to all the terms of the licence https://creativecommons.org/licences/by/3.0

Although reasonable endeavours have been taken to obtain all necessary permissions from third parties to include their copyrighted content within this article, their full citation and copyright line may not be present in this Accepted Manuscript version. Before using any content from this article, please refer to the Version of Record on IOPscience once published for full citation and copyright details, as permissions may be required. All third party content is fully copyright protected and is not published on a gold open access basis under a CC BY licence, unless that is specifically stated in the figure caption in the Version of Record.

View the article online for updates and enhancements. 


\title{
Can ionic concentration changes due to mechanical deformation be responsible for the neurostimulation caused by focused ultrasound? A simulation study.
}

\author{
Vladimir Filkin ${ }^{1}$, Igor Kuznetsov ${ }^{1}$, Olga Antonova ${ }^{1}$, llya Tarotinn ${ }^{2}$, Alexander Nemov ${ }^{1}$, and Kirill \\ Aristovich $^{2}$ \\ ${ }^{1}$ Higher School of Mechanics and Control, Peter the Great St. Petersburg Polytechnic University, Russia \\ ${ }^{2}$ Department of Medical Physics and Biomedical Engineering, University College London, UK
}

Abstract

Objective

Ultrasound stimulation is an emerging neuromodulation technique, for which the exact mechanism of action is still unknown. Despite the number of hypotheses such as mechanosensitive ion channels and intermembrane cavitation, they fail to explain all of the observed experimental effects. Here we are investigating the ionic concentration change as a prime mechanism for the neurostimulation by the ultrasound.

\section{Approach}

We derive the direct analytical relationship between the mechanical deformations in the tissue and the electric boundary conditions for the cable theory equations and solve them for two types of neuronal axon models: Hodgkin-Huxley and C-fibre. We detect the activation thresholds for a variety of ultrasound stimulation cases including continuous and pulsed ultrasound and estimate the mechanical deformations required for reaching the thresholds and generating action potentials.

Main results

We note that the proposed mechanism strongly depends on the mechanical properties of the neural tissues, which at the moment cannot be located in literature with the required certainty. We conclude that given certain common linear assumptions, this mechanism alone cannot cause significant effects and be responsible for neurostimulation. However, we also conclude that if the lower estimation of mechanical properties of neural tissues in literature is true, or if the normal cavitation occurs during the ultrasound stimulation, the proposed mechanism can be a prime cause for the generation of action potentials.

Significance

The approach allows prediction and modelling of most observed experimental effects, including the probabilistic ones, without the need for any extra physical effects or additional parameters. 


\section{Introduction}

Ultrasound neurostimulation is a relatively new neuromodulation technique, which has shown very promising results in targeted neurostimulation in the brain and peripheral nerves [1], [2]. It has the potential to overcome limitations of other existing neurostimulation methods, such as electrical, magnetic, and optical methods, and have the advantages of being completely non-invasive, portable and relatively cheap. The significant problem with the method's applicability is the lack of understanding of its underlying fundamental mechanism, which renders it impossible to optimise and fine-tune the technique, predict the outcome, and further develop the capabilities of the method [3], [4]. As a result, most of the research in this area is done by trial-and-error and the field, in general, contains very scattered, sometimes contradicting results [5]-[8].

Among the existing hypotheses the most promising are mechanosensitive ion channels [9], [10], intramembrane cavitation [11]-[13], sonoporation [14], [15] and thermal effects [3], [4], [15]. Mechanosensitive ion channels can be opened by mechanical deformations of a lipid bilayer which causes the influx of ions under the electrochemical gradient and generates the action potential (AP) in an axon. Mechanosensitive channels were proven to exist; this hypothesis is one of the most studied today, but its role in the ultrasound neurostimulation process remains unclear [16].

The most well-developed hypothesis is currently the theory of intramembrane cavitation. The idea of the method is based on the formation of the bubbles within the layers of a lipid bilayer (intramembrane). The bubbles cause geometrical changes which lead to a change in membrane capacitance. As an example, the model allows the prediction of how the experimentally observed efficacy of cortical ultrasonic stimulation depends on stimulation parameters [11]. However, it is still unclear whether the intramembrane cavitation occurs during an ultrasound stimulation. Some papers demonstrate the evidence in favour of this (e.g. [3], [5]), whereas others demonstrate the opposite ([6]-[8]) and present the case against the bubble formation.

Another existing hypothesis, sonoporation, suggests that ultrasound opens pores, which allows ions to move through a membrane similar to how it happens in the case of mechanosensitive ion channels. This mechanism was observed and studied [17], [18], but its role in neurostimulation remains unclear. One of the concerns is that sonoporation is associated with the presence of microbubbles, which increase the probability of injuries. Such conditions are usually avoided in experimental studies, but ultrasound stimulation is still observed [3].

Finally, several thermal effects occur in the tissue under the ultrasound stimulation, which may play a role in the activation mechanism. One of the essential effects is the temperature increase, which causes increased ionic mobility and changes in the diffusion process. Another hypothesis introduces thermosensitive ion channels, which can open with the increase in temperature. These effects are responsible for the infrared neural stimulation effect, however, there is no evidence that this mechanism plays a major role in an ultrasound neurostimulation process [19], [20].

In summary, none of the existing hypotheses for the mechanism of ultrasound stimulation can solely describe the full set of effects observed experimentally. As a result, there is no fundamental model for this mechanism that could predict and inform novel techniques required for non-invasive medical brain and nerve stimulation.

None of the above hypotheses takes into account the ionic concentration changes inside and around an axon. Existing literature [21], [22] and basic physical assumptions suggest that it could potentially be an important effect crucial for the process of ultrasound neurostimulation. There are two types of possible concentration change effects. The first one is the local increase in diffusion 
intensity caused by perturbation of steady ion distributions. That is, when a membrane and fluid vibrate, micro streams can occur and these streams can destroy a steady-state distribution of ions by mixing the ion-containing fluid up [21]. This effect however should have a cumulative and secondary nature and therefore could not be responsible for, for example, short-pulsed ultrasound neurostimulation.

The second effect is the changes in ionic concentration caused by the volumetric deformation of tissue. In this case, the ultrasound stimulation affects an axon by volumetric contraction or expansion of the fluid and membrane. If a small piece of the matter contracts quick enough with respect to the ionic motion, the number of ions within the given piece of the matter does not change and that leads to changes in the ionic concentration as well as in mass density. This effect is likely to be the cause of short-term processes which lead to the AP generation in the neuronal membrane. This was the focus of the current study.

The presented approach is based on the mechanical and electrochemical characteristics which are very similar for all types of neuronal axons, so the work was not aimed at targeting any specific region of the human neural system. However, the models of an axon that are used in the investigation correlate to the peripheral nervous system. Specifically, we were interested in the model of the Cfibre and Hodgkin-Huxley $(\mathrm{HH})$ model. The former was originally developed for the mammalian nociceptor axon, but also closely resembles the C-fibre axons, predominately populated within the vagus nerve, particularly the subdiaphragmatic vagus nerve [23]. The $\mathrm{HH}$ model represents a giant squid axon and is the most common model of unmyelinated fibre used in literature to investigate qualitative effects in peripheral and central NS [24].

Prior ex-vivo and in-vivo experimental studies show the possibility of neurostimulation of a peripheral nervous system, which was shown to be adequately represented by the above models. Both pulsed and continuous stimulation can be used for AP generation, although the pulsed scenario seems to be used more broadly [4]. To be more specific, there are several studies on ultrasound and mechanical stimulations of vagus nerves, C-fibres and giant squid axons in the literature [25]-[27]. Pulsed ultrasound modulation of a vagus nerve with a frequency close to $1 \mathrm{MHz}$ was shown to be effective and the effect increases with an increase of intensity [27]. On the other hand, several studies show that the vagus nerve can also be stimulated by low-intensity ultrasound [28], [29]. These studies also show that ultrasound stimulation affects the vagus nerve in the same way as electrical vagus nerve stimulation, which was proven to be an effective method for the treatment of drug-resistant disorders [30].

The purpose of the study was to investigate the feasibility of the concentration changes to cause the generation of action potentials during the ultrasound stimulation using the models of unmyelinated fibres, and to analyse the experimental implications and predictions. The specific questions were:

i. Can this mechanism be responsible for AP generation by ultrasound stimulation?

ii. What are the implications for the experimental predictions?

\section{Methods}

a. Study design.

We conducted a set of computational experiments to investigate the feasibility of the ultrasound-induced ionic concentration to cause the generation of AP in neuronal axons. We used Hodgkin-Huxley [31] and C-fibre [32] models of an axon, which were electrically coupled to the 
extracellular space through 3D FEM. We established a simple analytical relationship between ultrasound-induced geometric mechanical strain [33], [34] and the boundary conditions for purely electric axonal models - the second derivative of the potential with respect to a coordinate along the main axis of the axon (activating function). Finally, we conducted a series of computational experiments varying different parameters of ultrasound stimulation (amplitude, frequency, ultrasound stop phase, pulse width, and inter-stimulus interval), and observed the generation of AP propagating along the axon. We summarized and qualitatively assessed the results and made conclusions about the size of the effect, the feasibility of it being the prime mechanism for AP generation, and how well the observed qualitative effects could match existing experimental data.

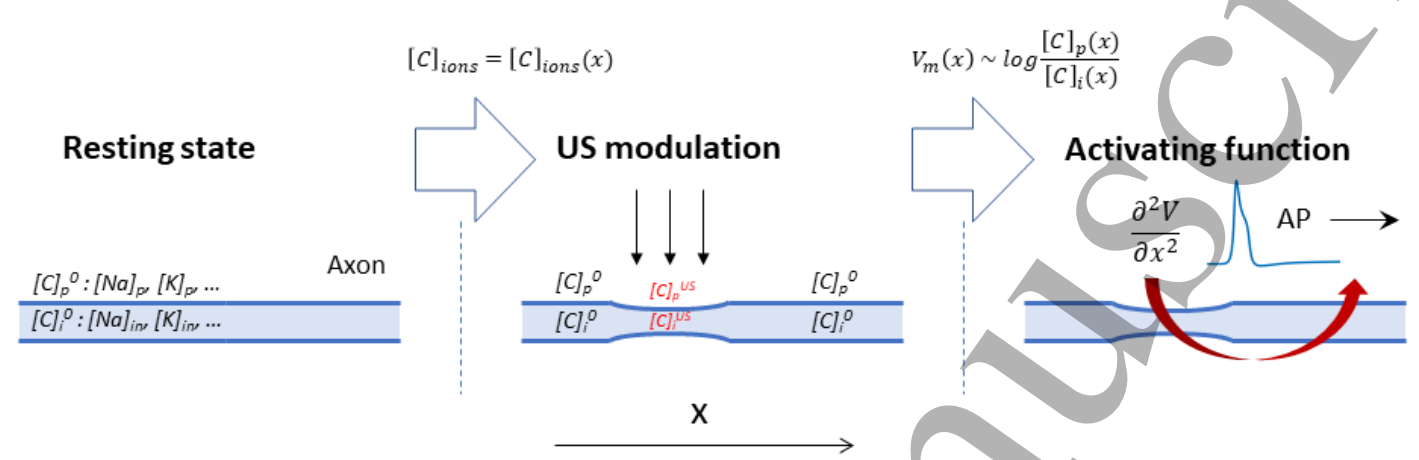

Figure 1. Schematic representation of the ultrasound stimulation mechanism. In the resting state, concentrations of ions inside and outside of the axon are constant along its length ("Resting state"). During ultrasound stimulation, the volumes of intracellular and periaxonal spaces change in the area of stimulation, and so do concentrations of the ions in this area ("ultrasound modulation"). Since the membrane potential is based on these concentrations, it will be variable with the coordinate along the axis of the fibre. This, therefore, leads to the initiation of the activating function defined as a second derivative of the external potential along the fibre. The activating function leads to the action potential generation ("Activating function").

b. Mechanical deformations

Ultrasound is a wave of changing density and pressure where changes in density can be described by mechanical deformations (strains). Although we aim to model the focused ultrasound beam, a plane wave with the axis parallel to the axis of an axon was considered in this study (i.e., the wave propagated along the axon). This assumption allowed us to simplify analytical equations without loss of generality. Axons angled to the plane wave could be represented in the same way with addition of a scaling factor obtained using the angle between the plane wave axis to the axonal axis. The only guiding parameter here was the bulk strain, which did not significantly depend on this angle. So, a wave propagating in any direction would influence a section of the axon in a similar way. We assumed that deformation had a sinusoidal form along the axonal axis, with constant amplitude, which is in good agreement with existing literature [35]. We also assumed that the changes in ionic concentration were proportional to the changes in density and that ionic motion was several orders of magnitude lower than the wave speed [22], thus also having a sinusoidal spatial waveform.

Under the mentioned assumptions, mechanical deformations (strains) are periodical in time and space. Two approaches were chosen to approximate them: a moving wave (1), which modelled the continuous ultrasound, and the standing wave (2), which better described the focused ultrasound:

$$
\varepsilon=A \sin \left(2 \pi f\left(t-\frac{x}{c}\right)\right)
$$




$$
\varepsilon=A \sin (2 \pi f t) \sin \left(\frac{2 \pi x}{\frac{c}{f}}\right)
$$

where $\varepsilon$ - mechanical strain along the axis of ultrasound wave propagation, $t-$ time, $x$-axis coordinate of an axon, $f$-frequency, $c$ - the speed of sound in this medium, $A$-amplitude of the mechanical strain.

The only unknown parameter here is the strain amplitude $A$. Since the pressure in the tissue under ultrasound is known (it can reach $1 M P a$ and more), the strain amplitude could be found from constitutive equations governing the elastic behaviour of the media. We assume that the surrounding liquid is compressible and cannot pass through the membrane at all. Then, mechanical strain in the membrane in any direction is simply related to mechanical stress:

$$
\varepsilon=\frac{\sigma}{3 K}
$$

where $K$ is compressibility modulus (bulk modulus) of the media which includes both axonal membrane and surrounding liquid, and $\sigma$ is the average normal stress, which for the considered case of volumetric compression/expansion equals to the applied pressure with the opposite sign. However, the actual estimation of $K$ for a brain poses significant controversy in the literature. Some researchers claim that the bulk modulus of the brain tissue must be close to that of water, $K \approx 2 G P a$ [36]. Similar results can be obtained from the measurements of ultrasound propagation velocity [37]-[39], which under the assumption that elastic wave propagation speed $c=\sqrt{K / \rho}$, where $\rho$ - density of a medium, allows estimation of $K \approx 2.4 \mathrm{GPa}$. A usual acoustic pressure $\sigma=1 \mathrm{MPa}$ [3], [4], [27], [40] leads to the strain amplitudes of the order of $0.0001(0.01 \%)$. On the other hand, assuming that the brain tissue is a linear isotropic elastic material, one can use relationships between elastic constants $(K=$ $\left.\frac{E}{3(1-2 v)}\right)$. This approach gives us the much smaller value of $K$ of the brain tissue: $10 \mathrm{kPa}$ according to [41] and about $1 \mathrm{MPa}$ in [42]-[45]. In those cases, the same acoustic pressure $\sigma=1 \mathrm{MPa}$ leads to significantly higher strains up to $1(100 \%)$. Experimental and analytical estimations of deformations in the peripheral nervous system also show that the tissue can deform up to $1(100 \%)$ during the application of ultrasound [46], [47].

There are therefore several contradicting pieces of evidence that lead to almost opposite results with respect to mechanical deformations of the membrane and do not allow to definitively state the relationship between the acoustic pressure and the mechanical deformations induced by the ultrasound. Here the influence of the mechanical deformations on the electrical behaviour of the axon was investigated, irrespective of the acoustic pressure. The detailed implications of using different pressure-deformation models are then provided in the discussion.

c. Ultrasound-induced boundary conditions for the $\mathrm{HH}$ and the $\mathrm{C}$-fibre models

In this section, the analytical relationship between the ultrasound-induced mechanical deformation and electric cable theory boundary conditions (activating function) is delivered. The voltage across the membrane of the axon could be expressed using the charge and the membrane capacitance through the following equations:

$$
V_{m 0}=\frac{Q}{C_{m 0}}
$$




$$
V_{m}=\frac{Q}{C_{m}}
$$

Where $V_{m 0}$ - the resting potential of a membrane, $V_{m}$ - membrane potential, $Q$ - full charge, $C_{0 m}$ and $C_{m}$ - resting and changed capacitances.

There are two ways to express the changes to the voltage due to mechanical deformation. We considered an infinitely small cylindrical cut-off of the axon as shown in Figure 2. The rate of mechanical deformations considered in this study is much quicker than transmembrane ionic movement [48], therefore the total charge on both sides of the axon was assumed to be constant.

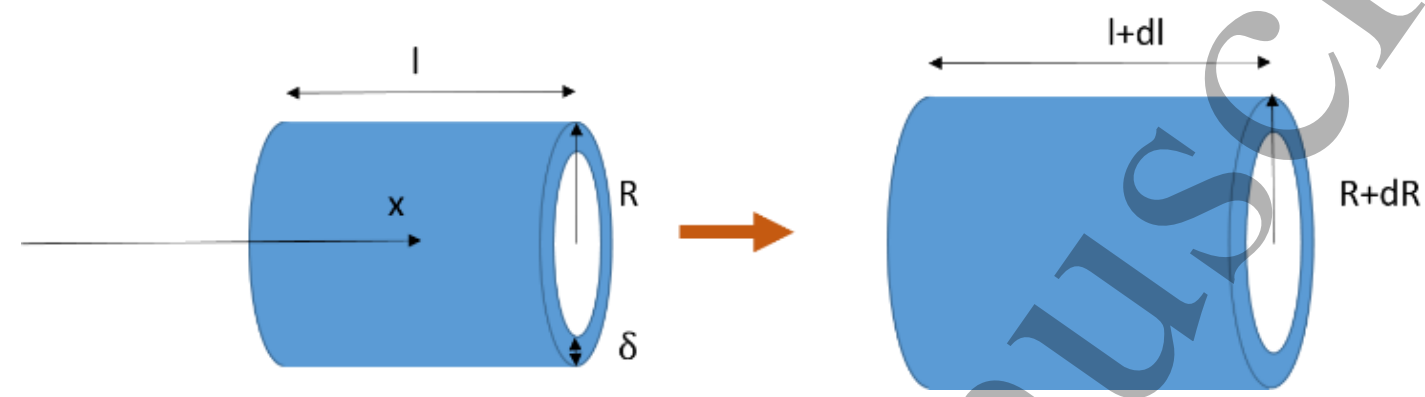

Figure 2. Schematic representation of an axon deformation.

The voltage across the membrane for the deformed state relative to the undeformed state can be expressed as a ratio of the cylindrical membrane capacitors, where we assumed that the membrane thickness did not change:

$$
\frac{V_{m}(x)}{V_{m 0}}=\frac{C_{m 0}}{C_{m}(x)}=\frac{A_{m 0}}{A_{m}(x)}=\frac{l 2 \pi R}{2 \pi(l+d l)(R+d R)}=\frac{1}{(1+\varepsilon(x))^{2}} \sim 1-2 \varepsilon(x)
$$

Where $A_{0 m}$ and $A_{m}$ are areas of a membrane conductor, $R$ - radius of a membrane, $d R-$ radius change due to ultrasound, $l$ - length of an axon, $d l$ - length change due to ultrasound, $\varepsilon-$ strain (deformation) $\left(\varepsilon=\frac{d R}{R}=\frac{d l}{l}\right)$. We considered the strain in the point to be uniform in an infinitely small volume around the segment. For the small strains $(\varepsilon(x) \ll 1)$ we can express $\frac{1}{(1+\varepsilon(x))^{2}}$ in a Taylor series as $1-2 \varepsilon(x)$.

The same final relationships can be also obtained by considering the infinitely small section of the membrane and calculating the change in concentration of the charge due to the volume changes, assuming the capacitance of the section stays the same. The second spatial derivative of external voltage along the axis of the axon, which is commonly called the activating function [49] can be expressed by the following set of equations according to the cable theory [50], [51]:

$$
\frac{\partial^{2} V_{e}}{\partial x^{2}}=-\left(1+\frac{R_{i}}{R_{e}}\right)^{-1} \frac{\partial^{2} V_{m}}{\partial x^{2}}
$$

Where $R_{i}$ and $R_{e}$ are electrical resistivities of intracellular and extracellular space respectively. Substituting the relationship (7) for the mechanical strains results in: 


$$
\frac{\partial^{2} V_{e}}{\partial x^{2}}=-\left(1+\frac{R_{i}}{R_{e}}\right)^{-1} \frac{\partial^{2}\left(V_{0} \frac{1}{(1+\varepsilon(x))^{2}}\right)}{\partial x^{2}} \approx-\left(1+\frac{R_{i}}{R_{e}}\right)^{-1} \frac{\partial^{2}\left(V_{0}(1-2 \varepsilon(x))\right)}{\partial x^{2}}
$$

During the ultrasound propagation, the strain is $\varepsilon_{0} \sin \left(\frac{2 \pi x}{\lambda}\right)$ as in $(1,2)$, where $\varepsilon_{0}$ is a strain amplitude, and $\lambda$ is a spatial wavelength of the ultrasonic wave. Even focused ultrasound deformations can be approximated by sinusoidal function [35]. In this case, the equation for the activating function takes the form:

$$
\frac{\partial^{2} V_{e}}{\partial x^{2}}=-2 \varepsilon_{0}\left(\frac{2 \pi}{\lambda}\right)^{2} V_{0}\left(1+\frac{R_{i}}{R_{e}}\right)^{-1} \sin \left(\frac{2 \pi x}{\lambda}\right)
$$

Assuming that $\frac{\partial^{2} V_{e}}{\partial x^{2}}=A_{V} \sin \left(\frac{2 \pi x}{\lambda}\right)$, we can get an amplitude:

$$
A_{V}=-2 \varepsilon_{0} V_{0}\left(\frac{2 \pi}{\lambda}\right)^{2}\left(1+\frac{R_{i}}{R_{e}}\right)^{-1}
$$

For $\lambda=0.1 \mathrm{~cm}, R_{i}=0.05 \mathrm{k} \Omega \mathrm{cm}(\mathrm{HH}), V_{0}=60 \mathrm{mV}, R_{i}=0.0354 \mathrm{k} \Omega \mathrm{cm}$ (C-Fibre) and $R_{e}=$ $0.1 \mathrm{k} \Omega \mathrm{cm}$ we have $A_{V} \approx 1.4 \cdot 10^{5} \varepsilon_{0} \frac{\mathrm{mV}}{\mathrm{cm}^{2}}$ for the $\mathrm{HH}$ model and $A_{V} \approx 1.6 \cdot 10^{5} \varepsilon_{0} \frac{\mathrm{mV}}{\mathrm{cm}^{2}}$ for C-Fibre model. The above relationships allow us to connect the amplitude of strain to the amplitude of activating function that we apply as a boundary condition to the simulated axon.

If strains are not small (i.e. higher than $0.01(1 \%)$ ), we have:

$$
\begin{gathered}
\frac{\partial^{2} V_{e}}{\partial x^{2}}=-\left(1+\frac{\rho_{i}}{\rho_{e}}\right)^{-1} \frac{\partial^{2}\left(V_{0} \frac{1}{\left(1+\varepsilon_{0} \sin \left(\frac{2 \pi x}{\lambda}\right)\right)^{2}}\right)}{\partial x^{2}} \\
\frac{\partial^{2} V_{e}}{\partial x^{2}}=-8\left(1+\frac{\rho_{i}}{\rho_{e}}\right)^{-1} \frac{V_{0} \varepsilon_{0} \pi^{2}}{\lambda^{2}}\left(\frac{3 \varepsilon_{0}\left(\cos \left(\frac{2 \pi}{\lambda} x\right)\right)^{2}}{\left(1+\varepsilon_{0} \sin \left(\frac{2 \pi}{\lambda} x\right)\right)^{4}}+\frac{\sin \left(\frac{2 \pi}{\lambda} x\right)}{\left(1+\varepsilon_{0} \sin \left(\frac{2 \pi}{\lambda} x\right)\right)^{3}}\right)
\end{gathered}
$$

This equation allows us to precisely calculate an amplitude of activating function for strains close to $1(100 \%)$ and can be solved numerically. For example, when $\varepsilon_{0}=0.5, \lambda=0.1 \mathrm{~cm}, R_{i}=$ $0.05 \mathrm{k} \Omega \mathrm{cm}(\mathrm{HH}), R_{i}=0.0354 \mathrm{k} \Omega \mathrm{cm}$ (C-Fibre) and $R_{e}=0.1 \mathrm{k} \Omega \mathrm{cm}$ we have $A_{V} \approx 2 \cdot 10^{6} \frac{\mathrm{mV}}{\mathrm{cm}^{2}}$ for HH model and $A_{V} \approx 2.2 \cdot 10^{6} \frac{\mathrm{mV}}{\mathrm{cm}^{2}}$ for the C-Fibre model. Similar, for $\varepsilon_{0}=0.9$, we have $A_{V} \approx 10^{8} \frac{\mathrm{mV}}{\mathrm{cm}^{2}}$ for $\mathrm{HH}$ model and $A_{V} \approx 1.1 \cdot 10^{8} \frac{\mathrm{mV}}{\mathrm{cm}^{2}}$ for C-Fibre model assuming the same parameters.

The above expressions show that the ultrasound stimulation could be treated based on the same principles as the application of the electric current and its effect could be modelled using the same simulation concepts. 
There are two main forms of activating functions which we used in our study, simulating the moving and standing waves of ultrasound respectively, as per (1) and (2), and assuming that there are no non-linear and transient effects:

$$
\begin{gathered}
f_{a c t}(x, t)=\frac{\partial^{2} V_{e}}{\partial x^{2}}=f_{a c t} \sin \left(2 \pi f\left(t-\frac{x}{c}\right)\right) \\
f_{a c t}(x, t)=\frac{\partial^{2} V_{e}}{\partial x^{2}}=f_{a c t_{0}} \sin (2 \pi f t) \sin \left(\frac{2 \pi x}{\frac{c}{f}}\right)
\end{gathered}
$$

d. The models of the nerve fibres

Two types of unmyelinated nerve fibres with active ion channels were simulated in this study. The first type was based on the Hodgkin-Huxley model of the giant axon of the squid (HH model) [31], which consisted of three types of ion channels - active sodium and potassium channels as well as passive leakage. The second type of fibre was a realistic mammalian $\mathrm{C}$ nociceptor with 10 active ion channels and voltage-dependent ions' concentrations [32]. This model was required to verify the qualitative results of the $\mathrm{HH}$ model and more accurately predict the quantitative values of the considered parameters, as well as give direct relevance to the obtained results. The C-fibre model closely resembles the fibres that can be found in the vagus nerve, stimulation of which is the prime interest of the scientific community for the treatment of a wide variety of drug-resistant disorders [23]. These two models were used in this study to allow us both to investigate general and very common reliable model $(\mathrm{HH})$ and to check the translation ability of the approach, and the validity of the results on a highly nonlinear detailed system (C-fibre).

In the models, fibres were represented as 1-dimensional cables placed in a cylindrical extracellular space simulated using the FEM approach in COMSOL Multiphysics software, as in [52]. In this study, bi-directional coupling of the fibres and extracellular space was omitted since only the effect of the external electrical and acoustic fields on the fibres were investigated. The effect of the external electric field on the fibres was simulated using the concept of activating function [49]. All the parameters of the models and equations representing their electrical behaviour were derived from [52].

We have used a $25 \mathrm{~cm}$ and $7 \mathrm{~cm}$ long axons for the $\mathrm{HH}$ and $\mathrm{C}$-fibre models respectively. The speed of sound was chosen to be equal to $1500 \mathrm{~m} / \mathrm{s}$. The focus size depends on the wavelength (frequency). If the wavelength of the ultrasound was higher than $1 \mathrm{~cm}$, it was spaced on the area of a single wavelength; otherwise, it was spaced on the length of not less than $1 \mathrm{~cm}$. This assumption was made to allow one-to-one comparison between different parameters, which would not be possible using the focused approximation, as the focal region was blurred and changed with frequency. Prior calculations show no relationship between the number of waves spaced along an axon and the activation threshold. The possible reason for this is that all the effects investigated in our study occur at the edge of a stimulated zone.

The zone of stimulation was located $5 \mathrm{~cm}$ and $1.5 \mathrm{~cm}$ from the left end of the axon for the $\mathrm{HH}$ and C-fibre models respectively. Element size was selected in such a way that there were no less than 32 and 43 elements within one ultrasound wavelength for the $\mathrm{HH}$ and $\mathrm{C}$-fibre models respectively, to satisfy mesh convergence criteria. The adaptive time step was used for simulations: there were at least 20 temporal steps for one period of ultrasound stimulation. When ultrasound stimulation was stopped there were at least 1000 temporal steps per $1 \mathrm{~ms}$. 


\section{e. Simulation cases}

There were two different simulation cases in our investigation: continuous and pulsed (Figure $3 \mathrm{~A})$. In the continuous case, the ultrasound was applied without any break continuously during the whole simulation. Different durations were utilised, and both standing and moving waves were considered. The simulations lasted at least $1 \mathrm{~ms}$ after the ultrasound modulation so that the AP generation and propagation could be detected. Two main parameters were under investigation: ultrasound amplitude and frequency.

In the pulsed case, a short pulse of ultrasound was applied (Figure 3B). The pulse duration was chosen to be $5 * T$, where $T$ is a period of the ultrasound wave. The frequency was chosen to be $1 \mathrm{MHz}$ as in the majority of the existing experimental studies.

We have investigated the influence of the stop phase and ultrasound pulse durations on the AP generation. Stop phase is a phase at which the ultrasound pulse is stopped, ranged from 0 to $2 \pi$ as a phase of the sinusoidal function. In the finite element (FE) model it is a phase of an activating function which was applied at the last moment of each pulse for a pulsed ultrasound and at the last moment of a continuous ultrasound (which is equivalent to the physical meaning as far as the effect of ultrasound is approximated by the activating function).

A wide range of phases and amplitudes (Table 1) were studied to understand the relationship between parameters and the generation and propagation of the AP, measured at $1 \mathrm{~mm}$ left from the ultrasound stimulated area for Hodgkin-Huxley model and at $0.1 \mathrm{~mm}$ for C-Fibre model.

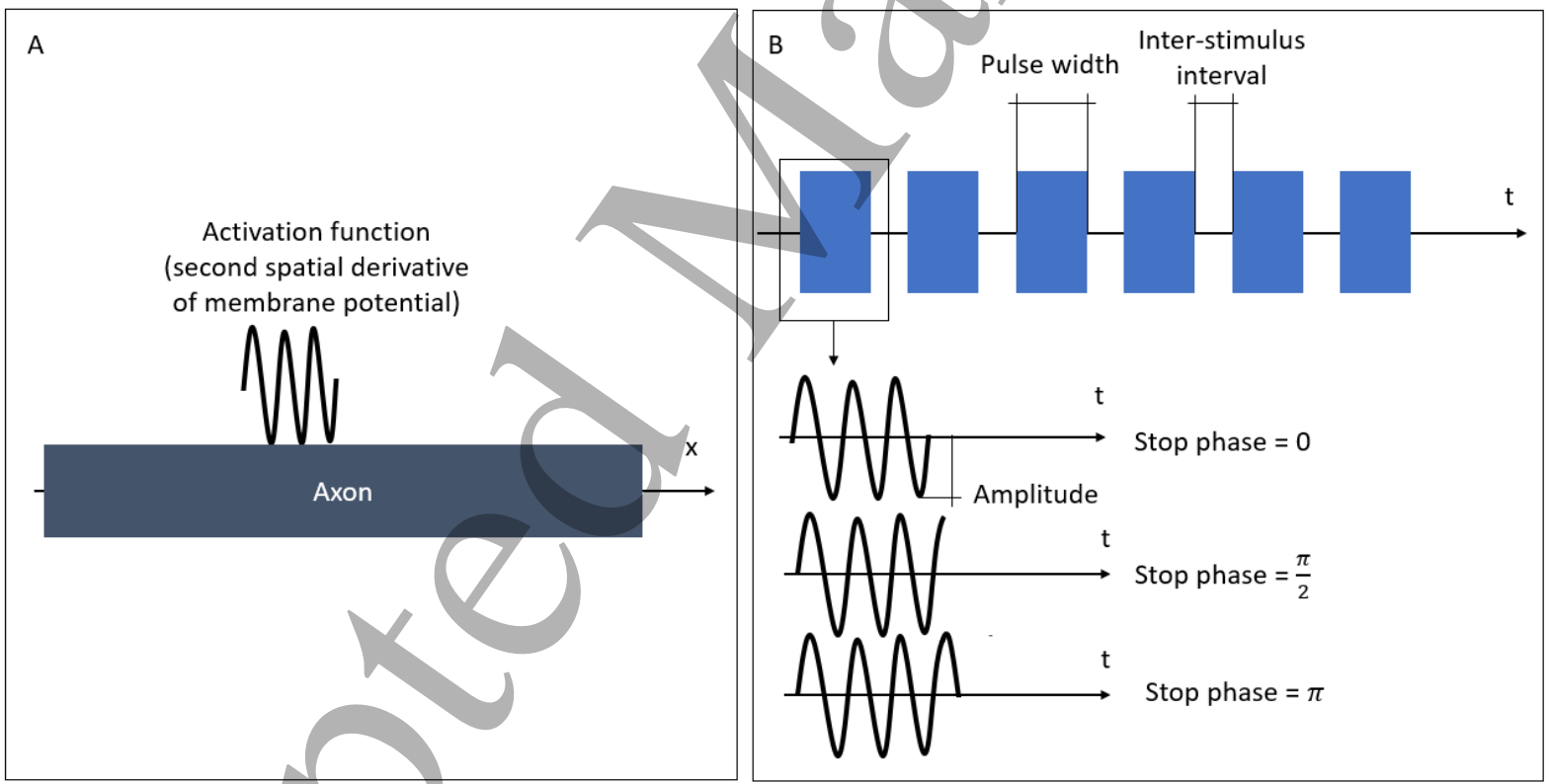

Figure 3. A) Activating function in space applied to an axon for both continuous and pulse cases B) Activating function in time for pulsed study. Pulses of the same specified duration are separated by inter-stimulus intervals (pauses). A pulse has an important parameter which is a stop phase. The stop phase is a phase of an activating function that was applied at the last moment of pulse duration.

Table 1. Parameter ranges for all studies

\begin{tabular}{|c|c|c|c|c|c|c|c|}
\hline \multirow{2}{*}{ Study } & \multicolumn{5}{|c|}{ Parameters } \\
\cline { 3 - 7 } & Model & $\begin{array}{c}\text { Frequency, } \\
M H z\end{array}$ & $\begin{array}{c}\text { Amplitude, } \\
\frac{m V}{c m^{2}}\end{array}$ & $\begin{array}{c}\text { Pulse width, } \\
\text { number } \\
\text { of periods }\end{array}$ & $\begin{array}{c}\text { Stop phase, } \\
\text { part a period }\end{array}$ & $\begin{array}{c}\text { Inter- } \\
\text { stimulus }\end{array}$ & $\begin{array}{c}\text { Number } \\
\text { of } \\
\text { pulses }\end{array}$ \\
\hline
\end{tabular}




\begin{tabular}{|c|c|c|c|c|c|c|c|}
\hline & & & & & & $\begin{array}{c}\text { interval, } \\
m s\end{array}$ & \\
\hline \multirow{2}{*}{ Continuous } & $\mathrm{HH}$ & $0.01-10$ & $10^{2}-10^{10}$ & & & \\
\hline & $\mathrm{C}-$ Fibre & $0.01-1$ & $10^{2}-10^{10}$ & & & \\
\hline $\begin{array}{c}\text { One pulse } \\
\text { (amplitude } \\
\text { and stop } \\
\text { phase) }\end{array}$ & $\mathrm{HH}$ & 1 & $10^{2}-10^{7}$ & 5 & $0-2 \pi$ & \\
\cline { 2 - 8 } & $\mathrm{C}-$ Fibre & 1 & $10^{7}-10^{9}$ & 5 & $0-2 \pi$ & \\
\hline $\begin{array}{c}\text { One pulse } \\
\text { (pulse } \\
\text { width) }\end{array}$ & $\mathrm{HH}$ & 1 & $10^{6}$ & $1-10000$ & $0, \frac{2 \pi}{5}, \pi, \frac{8 \pi}{5}$ & \\
\hline \multirow{2}{*}{\begin{tabular}{c} 
Pulsed \\
\cline { 2 - 8 }
\end{tabular}} & $\mathrm{C}-$ Fibre & 1 & $5 \cdot 10^{7}$ & $10-5000$ & $0, \pi$ & \\
\cline { 2 - 8 } & $\mathrm{C}-$ Fibre & 1 & $10^{6}$ & $1-1000$ & $0, \frac{3 \pi}{5}, \pi$ & 0.005 & $2-20$ \\
\hline $\begin{array}{c}\text { Pulsed } \\
\text { (random } \\
\text { phase) }\end{array}$ & $\mathrm{HH}$ & 1 & $10^{6}-10^{8}$ & 10,100 & $0, \pi$ & $0.01,0.05$ & $2,5,10$ \\
\hline & C-Fibre & $10^{6}$ & $10,50,100$ & random & 0.01 & $5,10,20$ \\
\hline
\end{tabular}

The influence of the pulse duration on the AP generation was investigated at frequencies of $1 \mathrm{MHz}$ and amplitudes of the activating function of $10^{6} \frac{\mathrm{mV}}{\mathrm{cm}^{2}}$ for $\mathrm{HH}$ model and $5 \cdot 10^{7} \frac{\mathrm{mV}}{\mathrm{cm}^{2}}$ for C-Fibre model (the amplitudes were determined as a threshold for the AP generation for the respective models).

A series of pulsed ultrasound was applied for close resemblance to experiments and real applications [3], [40], [53], [54]. Two new parameters were under investigation: number of pulses and duration of an inter-stimulus interval between pulses. Stop phase and duration of a pulse were also varied. Frequency and amplitude were chosen to be $1 \mathrm{MHz}$ and $10^{6} \frac{\mathrm{mV}}{\mathrm{cm}^{2}}$ for $\mathrm{HH}$ model and $1 \mathrm{MHz}$ with different amplitudes for C-Fibre model.

Finally, the probabilistic behaviour of ultrasound stimulation was investigated. Stop phases were randomly distributed from 0 to $2 \pi$, with the pulse length of 100 periods, and inter-stimulus interval of $0.01 \mathrm{~ms}$. Frequency and amplitude were $1 \mathrm{MHz}$ and $10^{6} \frac{\mathrm{mV}}{\mathrm{cm}^{2}}$ respectively. The study was conducted only for the $\mathrm{HH}$ model as it was not possible to compute for the C-fibre due to computational difficulties.

\section{Results}

a. Examples and description of AP generation and propagation using continuous and pulsed ultrasound

Both $\mathrm{HH}$ and C-fibre models demonstrated that the proposed approach could reliably generate initiation and propagation of the AP. The ultrasound-induced APs were $43.196 \mathrm{mV}$ and $26.6 \mathrm{mV}$ in membrane potential for $\mathrm{HH}$ and C-fibre model respectively, and their corresponding velocities were 1.5 and $0.08 \mathrm{~cm} / \mathrm{ms}$, which matches the literature for the typical slow-fibre action potentials [31], [52], [55]. The example studies demonstrated the stable AP initiation process and consequent propagation along the axon (Figure 4, Figure 5, Figure 6) at various ultrasound modulation parameters. 

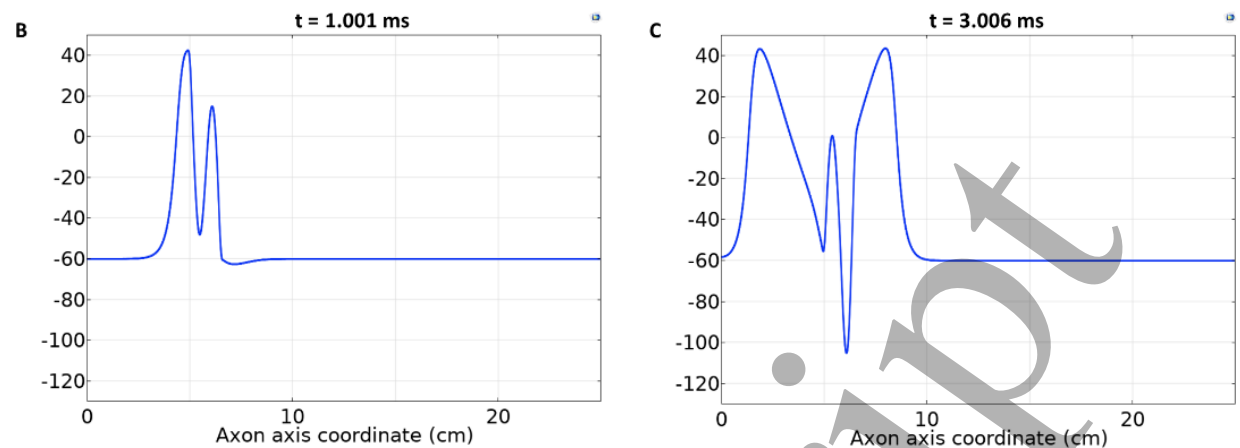

Figure 4. An example of the AP generation in the case of application a single-wave continuous ultrasound to the HH model. The ultrasound frequency was $0.1 \mathrm{MHz}$, the amplitude $-10^{5} \frac{\mathrm{mV}}{\mathrm{cm}^{2}}$. a) The initial phase at $t=0.103 \mathrm{~ms}, b$ ) The beginning of the AP generation at $t=1.001 \mathrm{~ms}, c)$ The AP propagation in both directions from the point of generation at $t=3.006 \mathrm{~ms}$. The resulting APs had membrane potentials equal $43.196 \mathrm{mV}$ and propagation velocities of $1.5 \mathrm{~cm} / \mathrm{ms}$, which matched the classic Hodgkin-Huxley model

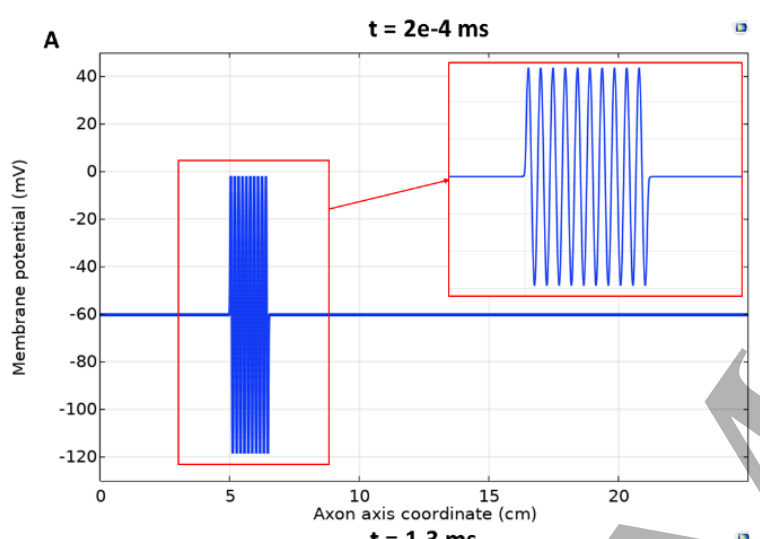

C

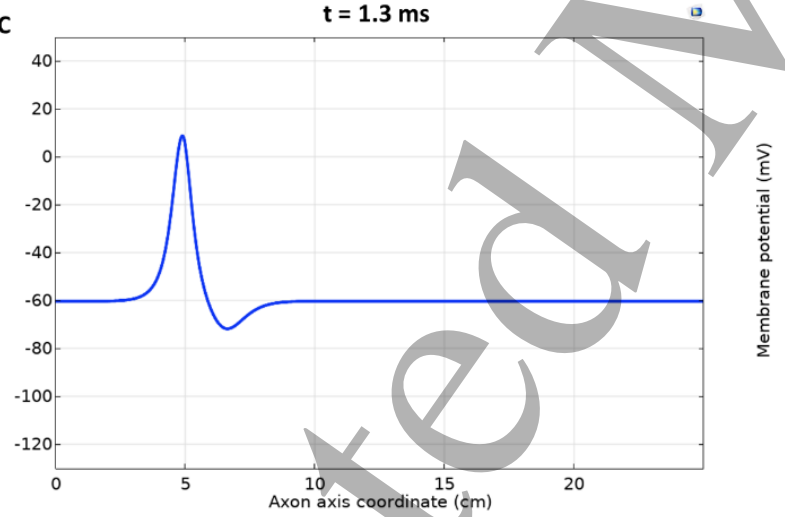

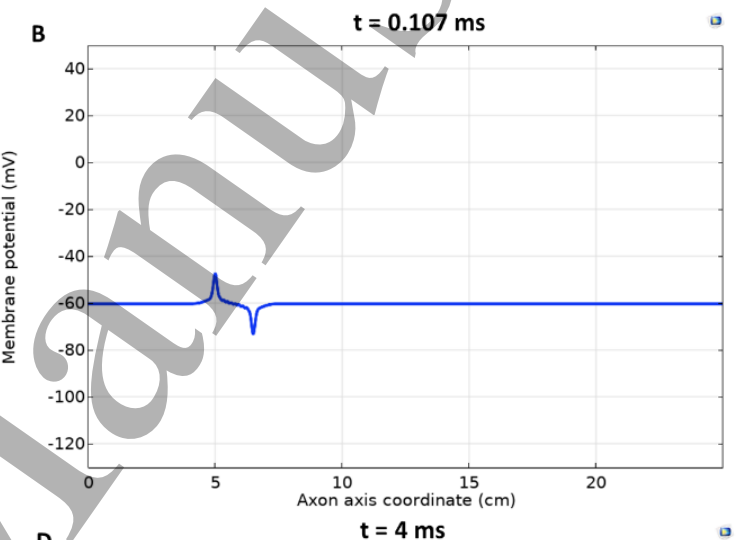

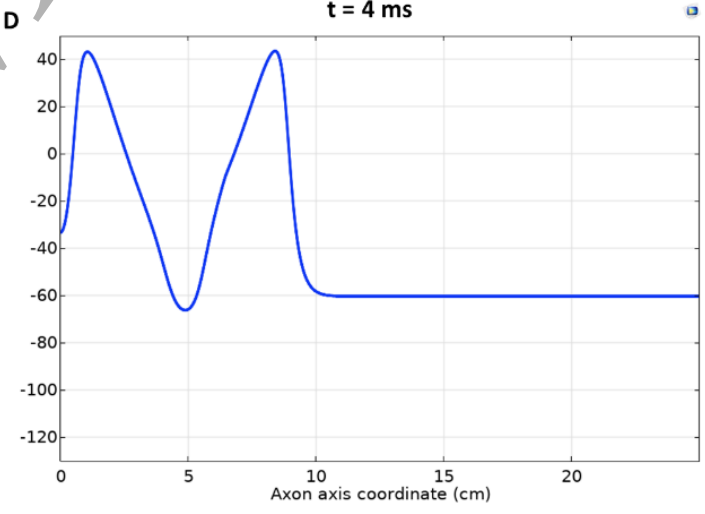

Figure 5. An example of the AP generation in the case of application of a pulsed ultrasound to the $\mathrm{HH}$ model. The ultrasound frequency was $1 \mathrm{MHz}$, the amplitude was $10^{6} \frac{\mathrm{mV}}{\mathrm{cm}^{2}}, 10$ pulses, 100 periods each pulse, $\pi$ stop phase, $0.01 \mathrm{~ms}$ inter-stimulus interval. a) The initial phase at $t=2 e-4 \mathrm{~ms}, b$ ) The inter-stimulus interval between pulses at $t=0.107 \mathrm{~ms} c$ ) The beginning of the AP generation at $t=1.3 \mathrm{~ms}$, d) The AP propagation in both directions from the point of generation at $t=4 \mathrm{~ms}$. The resulting APs had membrane potential equals to $43.196 \mathrm{mV}$ and propagation velocities of $1.5 \mathrm{~cm} / \mathrm{ms}$, which matches the classic $\mathrm{HH}$ model 

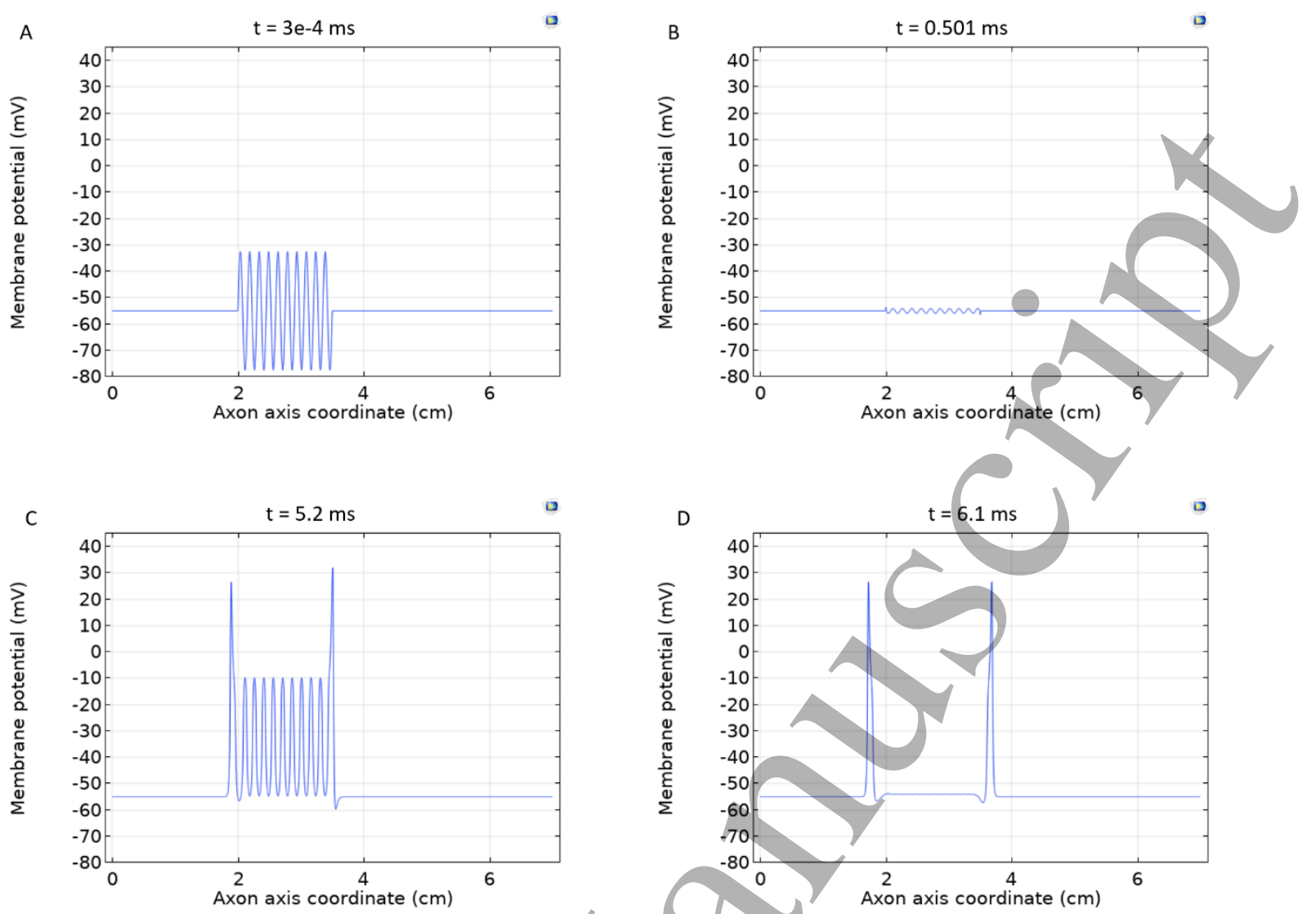

Figure 6. An example of the AP generation in the case of application of a pulsed ultrasound to the $C$ fibre model. The ultrasound frequency was $1 \mathrm{MHz}$, the amplitude was $10^{8} \frac{\mathrm{mV}}{\mathrm{cm}^{2}}, 10$ pulses, 100 periods each pulse, $\pi$ stop phase, 0.01 ms inter-stimulus interval. a) The initial phase at $t=3 e-$ $4 \mathrm{~ms}, b)$ The inter-stimulus interval between pulses at $\mathrm{t}=0.501 \mathrm{~ms} \mathrm{c}$ ) The beginning of the AP generation at $t=5.2 \mathrm{~ms}, d$ ) The AP propagation in both directions from the point of generation at $t=6.1 \mathrm{~ms}$. The resulting APs had membrane potential equals to $26.6 \mathrm{mV}$ and moving with the velocity of $0.08 \mathrm{~cm} / \mathrm{ms}$, which matches the classic AP

The comparison between standing and moving waves at frequencies of $100 \mathrm{kHz}$ with continuous ultrasound stimulation revealed that the standing wave has a lower threshold of AP generation by approximately half. At lower frequencies, this effect decreased so that below $10 \mathrm{kHz}$ there was no significant difference between standing and moving waves. Significantly higher amplitude was required for AP generation at higher frequencies in the case of moving wave, doubling the one for the standing wave at frequencies above $1 \mathrm{MHz}$. We were using a standing wave for the subsequent investigations as it was closer to the actual focused ultrasound used for neuromodulation.

b. Activation threshold in constant continuous ultrasound stimulation case

To find the activation threshold for the continuous ultrasound, different frequencies and amplitudes of activating function were applied for the whole duration of the simulation. The activating threshold varied with frequency (Figure 7) and was $10^{3} \frac{\mathrm{mV}}{\mathrm{cm}^{2}}$ for $10 \mathrm{kHz}$, and increased to $10^{8} \frac{\mathrm{mV}}{\mathrm{cm}^{2}}$ for $10 \mathrm{MHz}$ for the $\mathrm{HH}$ model, which corresponded to the mechanical strains of the axon by $0.68(68 \%)$ and $0.41(41 \%)$ respectively. In Figure 7 one can also find strain for each simulation case written in each cell. These strains show that an activation threshold in terms of strain is similar at all frequencies. However, there is a slight decrease of strains (from 0.68 to 0.41 ) for frequencies higher than $1 \mathrm{MHz}$. The same analysis for the C-fibre resulted in the threshold $10^{7} \frac{\mathrm{mV}}{\mathrm{cm}^{2}}$ for $10 \mathrm{kHz}$ and $10^{10} \frac{\mathrm{mV}}{\mathrm{cm}^{2}}$ for $1 \mathrm{MHz}$ 
respectively, which corresponded to 0.98 and 0.96 mechanical strain. The activation threshold in terms of strain is similar at all frequencies for the C-fibre model.
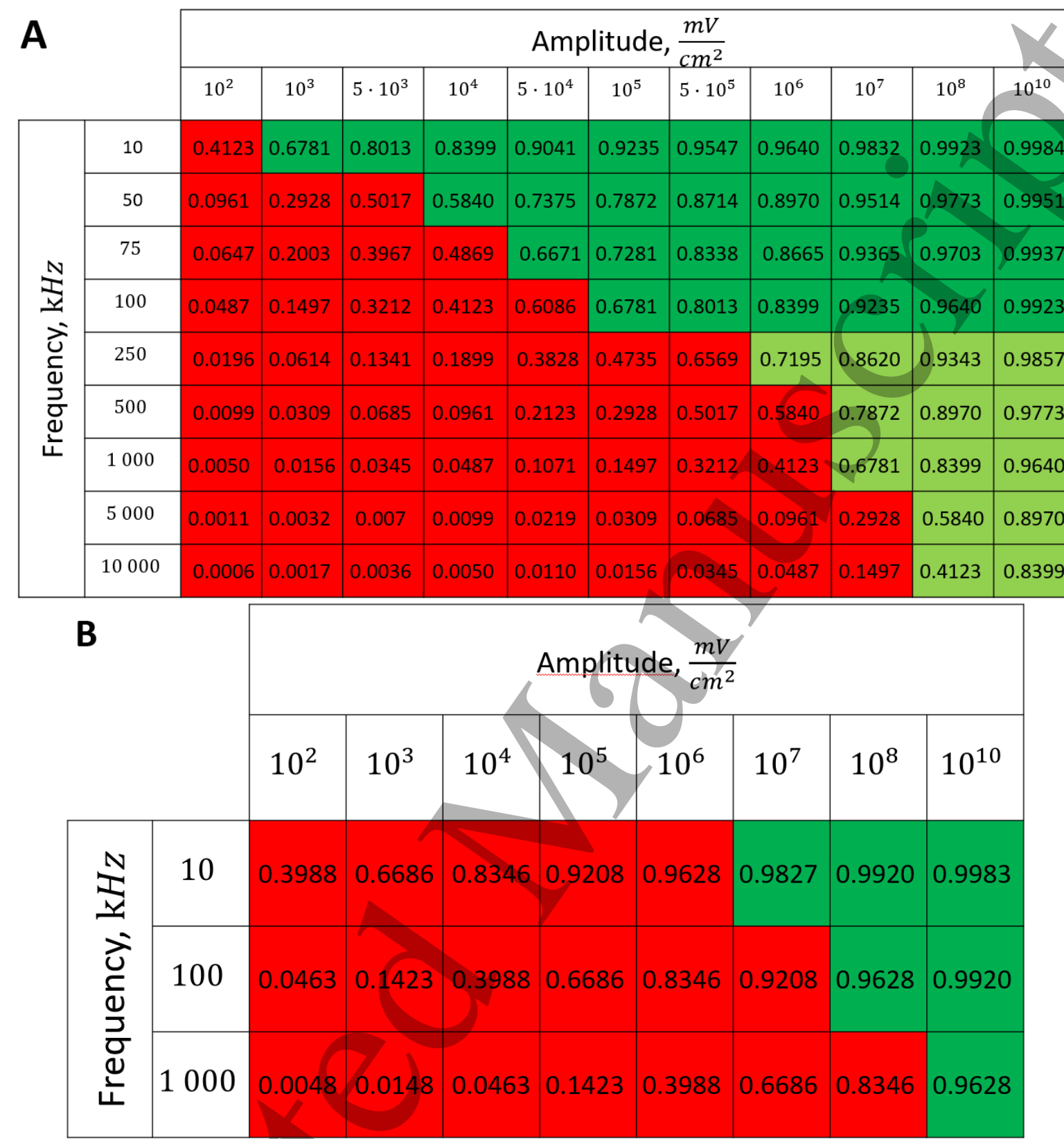

Figure 7. The summary of an axohal action potential generation test for a range of amplitudes and frequencies of a standing wave activating function boundary conditions (eq.12). Red colour means no AP was generated, dark green - AP was generated, light green - AP should be generated but the model does not allow to simulate the generation and propagation due to the numerical constraints. Each cell contains strain in an axon for an exact frequency and amplitude. The strains are estimated with equation (13). A) HH model. B) C-fibre model.

c. Activation threshold in pulsed ultrasound stimulation case

i. Phase - amplitude relationship for the single pulse stimulation

The activation threshold for the pulsed ultrasound was found for different stop phases and the length of the pulse of 5 periods $(5 \mu s)$ at the frequency of $1 \mathrm{MHz}$ (Figure 8A). AP was generated 
when the amplitudes of the activating function were larger than $5 \cdot 10^{6} \frac{\mathrm{mV}}{\mathrm{cm}^{2}}$ (0.61 strain). The stop phases between $\frac{2 \pi}{5}$ and $\frac{8 \pi}{5}$ (phases are expressed as a phase of sinusoidal function, see Figure 3 ) caused the highest membrane voltage change and subsequent AP generation. This suggested that the generation of AP was more sensitive to the total energy translated to the system rather than the maximum membrane potential (Figure 8 , red dots). The highest energy value translated to the system was at the phase of $\pi$ (the wave stopped just after the positive phase), which corresponded to the highest membrane voltage for all amplitudes. The amplitudes of the activating function below $10^{4} \frac{\mathrm{mV}}{\mathrm{cm}^{2}}$ (0.05 strain) did not significantly affect the membrane potential. For the C-fibre model the pattern was very similar (Figure 8). Phases between $\frac{3 \pi}{5}$ and $\frac{7 \pi}{5}$ were the most effective ones. The amplitudes below $10^{7} \frac{\mathrm{mV}}{\mathrm{cm}^{2}}$ (0.8 strain) did not significantly change the membrane voltage. 
A Maximal probe membrane potentials for stop phase/amplitude study

B

Maximal probe membrane potentials for
stop phase/amplitude study from stop phase

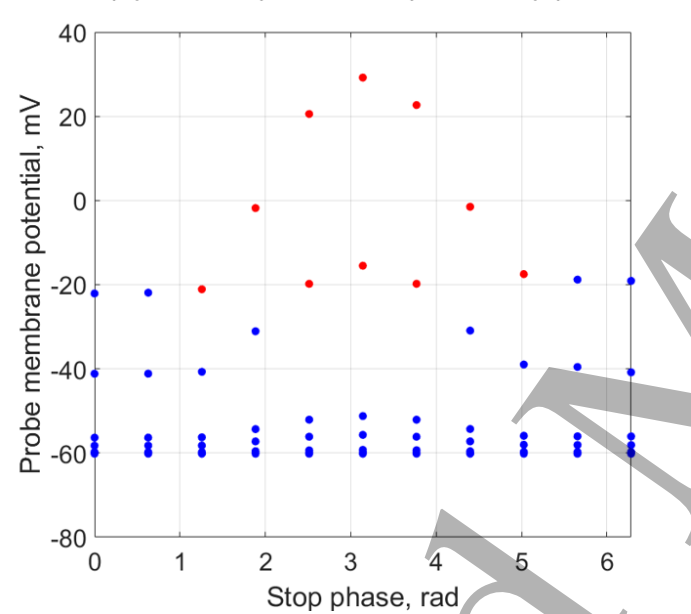

C
Maximal probe membrane potentials for
stop phase/amplitude study from amplitude

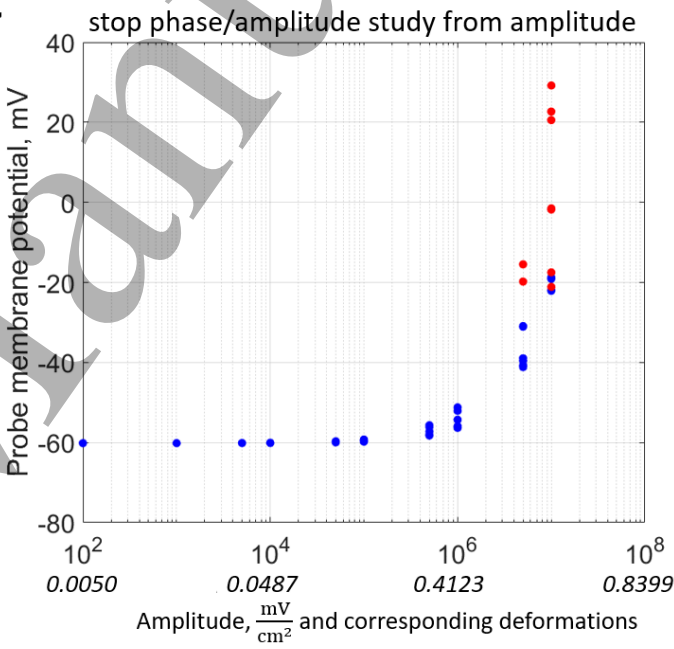

\begin{tabular}{|c|c|c|c|c|c|c|c|c|}
\hline & & \multicolumn{6}{|c|}{ Amplitude, $\frac{m V}{c m^{2}}$ and corresponding deformations } \\
\hline & & $\begin{array}{c}10^{7} \\
0.6686\end{array}$ & $\begin{array}{c}5 \cdot 10^{7} \\
0.7949\end{array}$ & $\begin{array}{c}10^{8}, \\
0.8346\end{array}$ & $\begin{array}{l}2 \cdot 10^{8}, \\
0.8671\end{array}$ & $\begin{array}{l}4 \cdot 10^{8}, \\
0.8936\end{array}$ & $\begin{array}{l}5 \cdot 10^{8}, \\
0.9009\end{array}$ & $\begin{array}{c}10^{9}, \\
0.9208\end{array}$ \\
\hline & & 55.01 & -55.01 & -54.97 & -54.95 & -54.92 & -54.58 & -54.46 \\
\hline & & 5.01 & -54.93 & -54.71 & -54.42 & -53.85 & -53.56 & -52.39 \\
\hline & & -54.84 & -54.01 & -53.08 & -51.31 & 26.74 & 26.74 & 26.74 \\
\hline & & 54.71 & -53.49 & -52.09 & 26.74 & 26.74 & 26.74 & 26.74 \\
\hline & & -54.79 & -54.01 & -53.04 & -51.45 & 26.74 & 26.74 & 26.74 \\
\hline & $9 \pi / 5$ & -55.01 & -54.8 & -54.71 & -54.42 & -53.95 & -53.62 & -52.42 \\
\hline & $2 \pi$ & -55.01 & -55.01 & -54.96 & -54.96 & -54.93 & -54.82 & -54.58 \\
\hline
\end{tabular}

Figure 8. The summary of an action potential generation for a range of stop phases and amplitudes of activating function. ultrasound was applied for the time of 5 periods and stopped at a specific stop phase (eq.12) at a frequency of $1 \mathrm{MHz} . A, B, C)$ Hodgkin-Huxley model. The maximal probe membrane potential recorded straight after stimulation at the probe location $1 \mathrm{~mm}$ to the left of the stimulated zone. Red dots indicate the generation of an action potential after the stimulation. 
D) C-Fibre model. Maximum membrane potential at a point $0.1 \mathrm{~mm}$ to the left from the stimulated zone straight after ultrasound stimulation. Red cells indicate the generation of an action potential after the stimulation.

ii. Pulse width analysis

Pulse widths and stop phases were varied at $1 \mathrm{MHz}$ with the activating function amplitudes $10^{6} \frac{\mathrm{mV}}{\mathrm{cm}^{2}}(0.41$ strain

) and $5 \cdot 10^{7} \frac{\mathrm{mV}}{\mathrm{cm}^{2}}$ (0.8 strain) for the $\mathrm{HH}$ and C-Fibre models respectively (Figure 9). The membrane potential in the $\mathrm{HH}$ model decreased with the increase of pulse width. As in the previous case, the stop-phase significantly affected the results with $\pi$ being optimal for the generation of the AP.

C-Fibre model behaved differently (Figure 9B). Membrane potential increased with the increase of a pulse width when the ultrasound was stopped at the zero stop phase. However, if the wave was stopped at $\pi$, the membrane potential decreased with the increase of pulse width. When pulse width reached the length of 1000 periods there was no significant difference in the output between the two stop phases.

A

Maximal probe membrane potentials

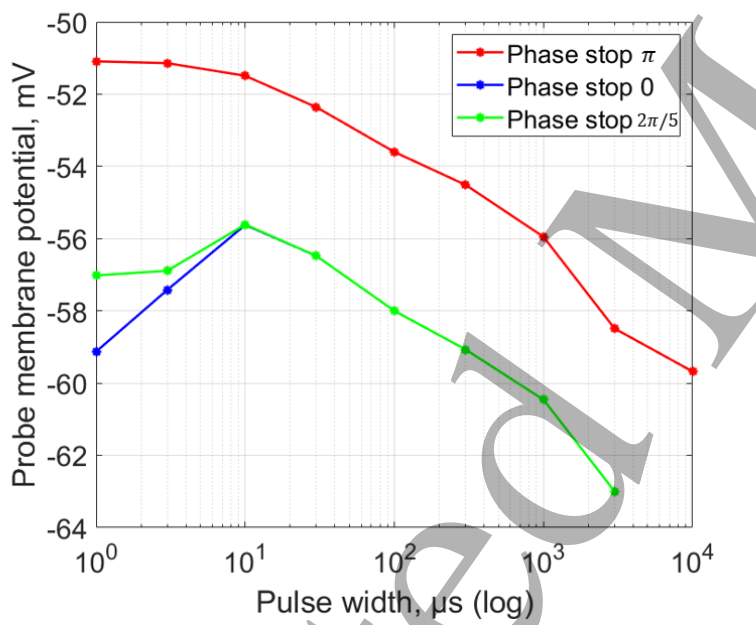

C Membrane potential at the point from
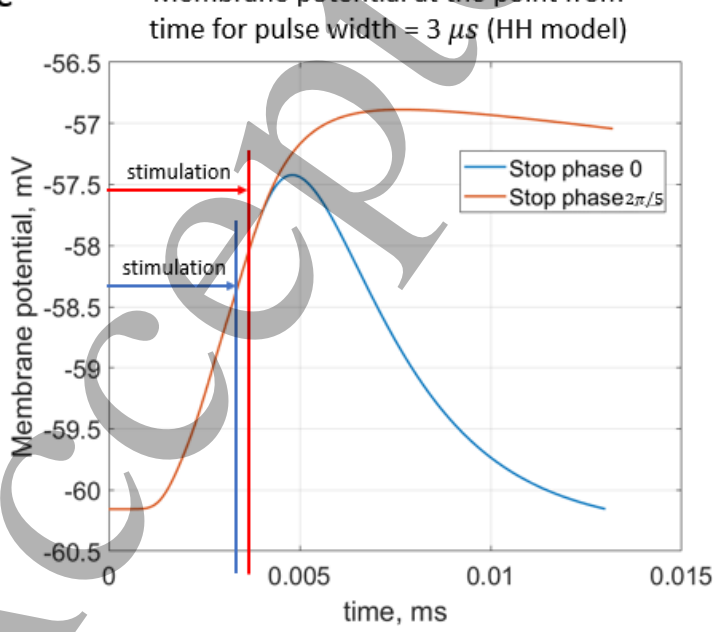

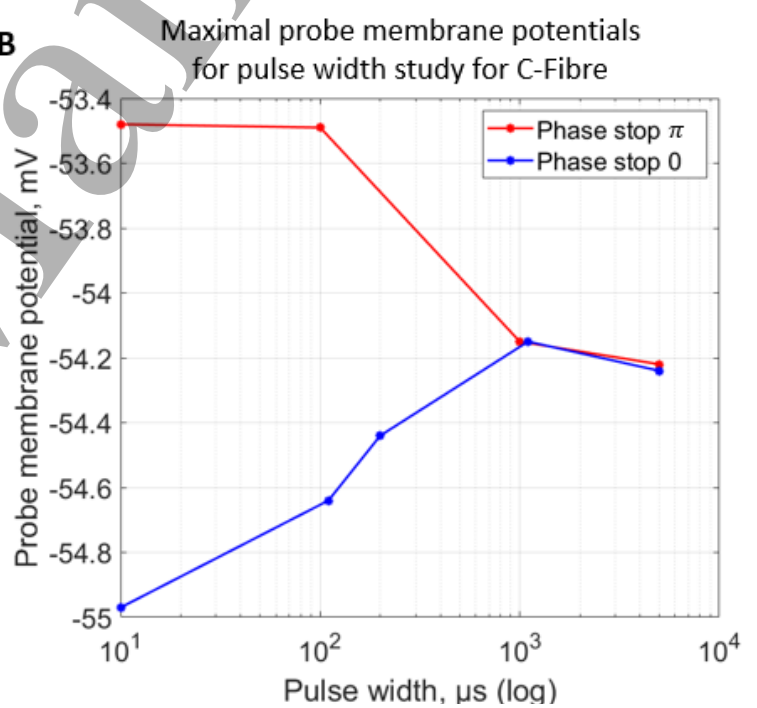

D Membrane potential at the point from time for

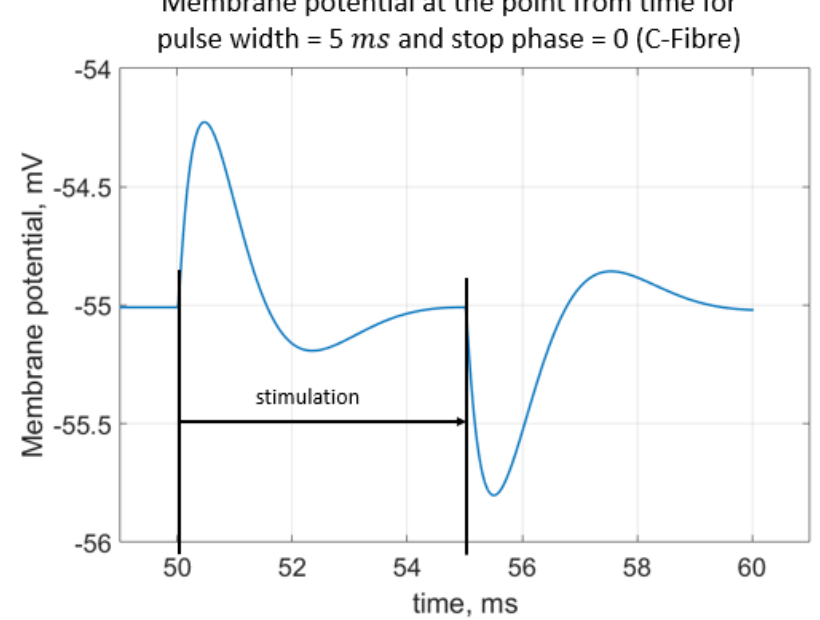



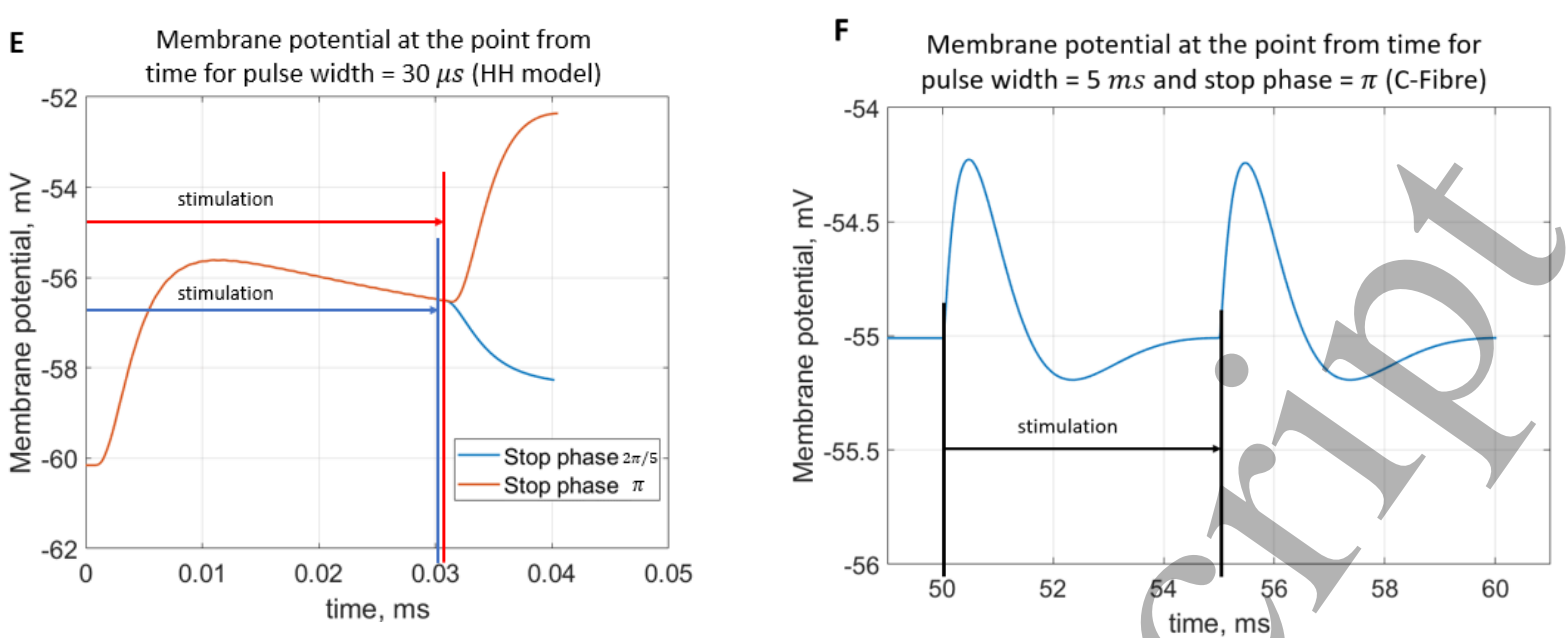

Figure 9. A, B) The summary of an action potential generation for a range of stop phases and durations of the activating function $A$ ) of $\mathrm{HH}$ model at the amplitude of $10^{6} \frac{\mathrm{mV}}{\mathrm{cm}^{2}}, B$ ) of C-Fibre model at the amplitude of $5 \cdot 10^{7} \frac{\mathrm{mV}}{\mathrm{cm}^{2}}$. C, D, E, F) Membrane potentials at a point $1 \mathrm{~mm}$ left from the stimulated zone with respect to time. C) $\mathrm{HH}$ model, pulse width is $3 \mu \mathrm{s}$, stop phases are 0 and $\left.\frac{2 \pi}{5}, D\right) C$-fibre, pulse width is $5 \mathrm{~ms}$, stop phase is 0, E) $\mathrm{HH}$ model pulse width is $30 \mu \mathrm{s}$, stop phases are $\frac{2 \pi}{5}$ and $\pi$, F) C-fibre, pulse width is $5 \mathrm{~ms}$, stop phase is $\pi$. The frequency was $1 \mathrm{MHz}$ in all cases.

iii. Analysis of the ultrasound modulation with pulse trains.

Here the activating thresholds and membrane potentials were investigated with respect to the number of pulses, pulse widths, stop phases, and interstimulus intervals (Table 1) for the pulsed ultrasound delivered using the series of pulses, or a train (Figure 10, Figure 11, Figure 12). A range of pulses of activating function with different widths was applied at $1 \mathrm{MHz}$. The amplitudes were $10^{6} \frac{\mathrm{mV}}{\mathrm{cm}^{2}}$ (0.41 strain) for the $\mathrm{HH}$ model and $10^{6}-10^{8} \frac{\mathrm{mV}}{\mathrm{cm}^{2}}(0.40-0.83$ strain) for C-Fibre model.

For the $\mathrm{HH}$ model, inter-stimulus interval duration did not significantly affect the membrane voltage changes, however shorter inter-stimulus intervals resulted in generally higher membrane potentials (Figure 10). The increase in the number of pulses was generally more effective in generating the action potential (Figure 11). Pulses for which the stop phase was 0 did not generate action potentials at all for our study parameters. The increase of a pulse width resulted in a significant decrease in the AP generation ability (Figure 11).

C-Fibre model behaved in a similar way (Figure 12). Amplitude had a major influence on the qualitative behaviour of the system. The number of pulses significantly changed the activating threshold at the amplitude of $5 \cdot 10^{7} \frac{\mathrm{mV}}{\mathrm{cm}^{2}}$ (0.8 strain) (Figure $12 \mathrm{E}, \mathrm{F}$ ).

Additional experiment with 0 stop phase showed that axon did not generate the AP at amplitude $1 e 8 \frac{\mathrm{mV}}{\mathrm{cm}^{2}}, 10$ pulses, $0.01 \mathrm{~ms}$ interstimulus interval and 100 periods $(100 \mu \mathrm{s})$ pulse width, whilst the AP was generated with a $\pi$ stop phase using the exact same parameters. 
A

40 ${ }_{41}$
A Pulse width $10 \mu s$, stop phase $\pi$ (HH model)

\begin{tabular}{|c|c|c|c|}
\cline { 2 - 4 } \multicolumn{1}{c|}{} & \multicolumn{3}{|c|}{ Inter-stimulus interval, $m s$} \\
\hline Pulses & 0.005 & 0.01 & 0.05 \\
\hline 2 & & & \\
\hline 5 & & & \\
\hline 10 & & & \\
\hline 20 & & & \\
\hline
\end{tabular}

B Membrane potential at the point from time for

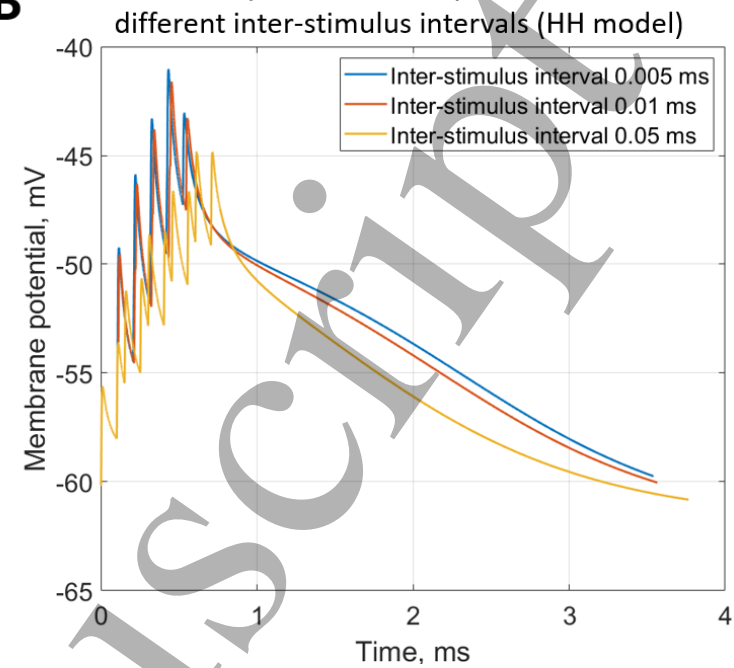

Figure 10. The summary of an axonal action potential generation for a range of number of pulses and inter-stimulus intervals of a standing wave pulsed activating function boundary conditions (eq.12). A) Red colour means no AP was generated, green - AP was generated. 10 periods $(\mu \mathrm{s})$ pulse width, $\pi$ stop phase, frequency $-1 \mathrm{MHz}^{2}$ amplitude $10^{6} \frac{\mathrm{mV}}{\mathrm{cm}^{2}}$. $B)$ Three different inter-stimulus intervals. Membrane potentials at a point $1 \mathrm{~mm}$ left from the stimulated zone on time are presented. Frequency $-1 \mathrm{MHz}$, amplitude $10^{6} \frac{\mathrm{mV}}{\mathrm{cm}^{2}}, 5$ pulses, pulse width $=100$ periods, stop phase $=\pi$ for each pulse.

Stop phase 0, inter-stimulus interval $0.01 \mathrm{~ms}$ Pulse width, $\mu$ s

\begin{tabular}{l|l|l|l}
1 & 10 & 100 & 1000 \\
\hline
\end{tabular}

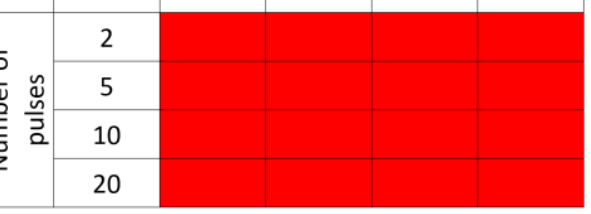

B Stop phase $3 \pi / 5$, inter-stimulus intêrval $0.01 \mathrm{~ms}$

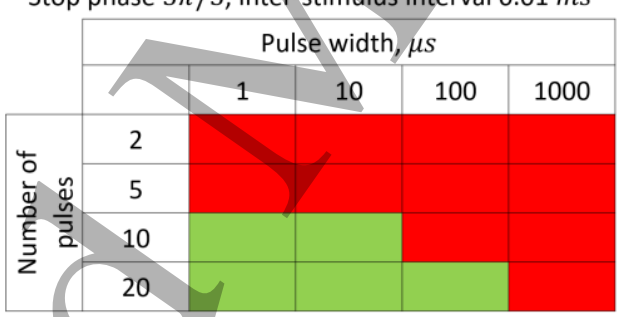

C Stop phase $\pi$, inter-stimulus interval $0.01 \mathrm{~ms}$

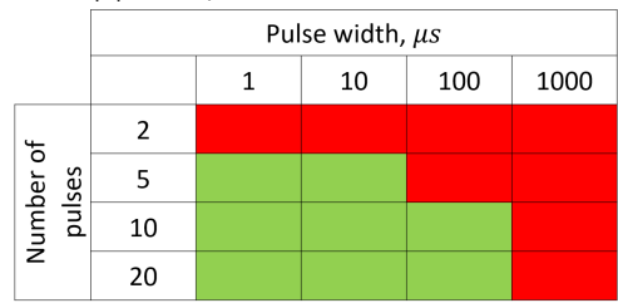
and pulse widths of a standing wave pulsed activating function boundary conditions (eq.12). Red colour means no AP was 43generated, green - AP was generated. Frequency $1 \mathrm{MHz}$, amplitude $10^{6} \frac{\mathrm{mV}}{\mathrm{cm}^{2}}$ (0.43 deformation), 0.01 ms interstimulus $45^{4}$ interval. A) stop phase $0, B$ ) stop phase $\frac{3 \pi}{5}$, C) stop phase $\pi$ 
Inter-stimulus interval $0.01 \mathrm{~ms}$, amplitude $10^{\wedge} 6 \mathrm{mV} / \mathrm{cm}^{2}$

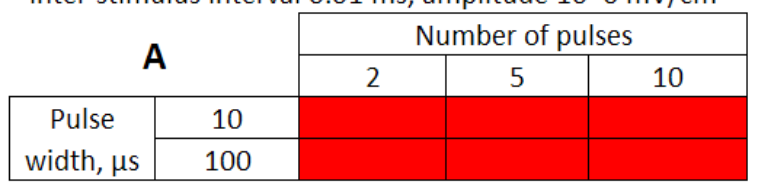

Inter-stimulus interval $0.01 \mathrm{~ms}$, amplitude $10^{\wedge} 7 \mathrm{mV} / \mathrm{cm}^{2}$

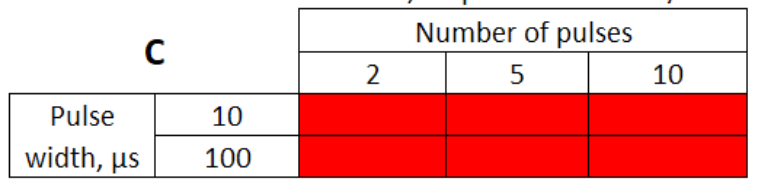

Inter-stimulus interval $0.01 \mathrm{~ms}$, amplitude $5^{*} 10^{\wedge} 7 \mathrm{mV} / \mathrm{cm}^{2}$

\begin{tabular}{|c|c|c|c|c|}
\cline { 3 - 5 } \multicolumn{2}{c|}{} & \multicolumn{3}{c|}{ Number of pulses } \\
\cline { 3 - 5 } \multicolumn{2}{c|}{} & 2 & 5 & 10 \\
\hline Pulse & 10 & & & \\
\cline { 3 - 5 } width, $\mu \mathrm{s}$ & 100 & & & \\
\hline
\end{tabular}

Inter-stimulus interval $0.01 \mathrm{~ms}$, amplitude $10^{\wedge} 8 \mathrm{mV} / \mathrm{cm}^{2}$

\begin{tabular}{|c|c|c|c|c|}
\cline { 3 - 5 } \multicolumn{2}{c|}{} & \multicolumn{3}{c|}{ Number of pulses } \\
\cline { 3 - 5 } \multicolumn{1}{c|}{ Pulse } & 10 & 2 & 5 & 10 \\
\cline { 3 - 5 } width, $\mu \mathrm{s}$ & 100 & & & \\
\hline
\end{tabular}

Inter-stimulus interval $0.05 \mathrm{~ms}$, amplitude $10^{\wedge} 6 \mathrm{mV} / \mathrm{cm}^{2}$

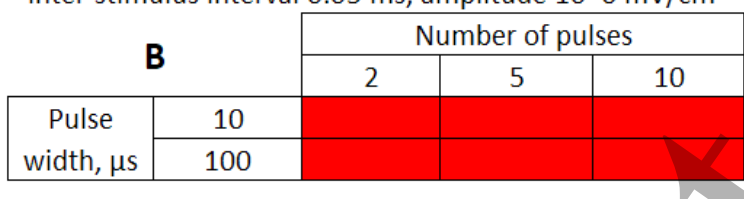

Inter-stimulus interval $0.05 \mathrm{~ms}$, amplitude $10^{\wedge} 7 \mathrm{mV} / \mathrm{cm}^{2}$

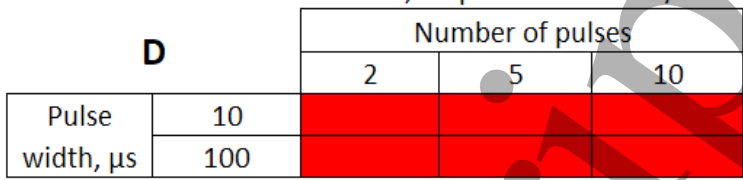

Inter-stimulus interval $0.05 \mathrm{~ms}$, amplitude $5 * 10^{\wedge} 7 \mathrm{mV} / \mathrm{cm}^{2}$

\begin{tabular}{|c|c|c|c|c|}
\hline \multirow{2}{*}{\multicolumn{2}{|c|}{$\mathbf{F}$}} & \multicolumn{3}{|c|}{ Number of pulses } \\
\hline & & 2 & 51 & 10 \\
\hline \multirow{2}{*}{$\begin{array}{c}\text { Pulse } \\
\text { width, } \mu \mathrm{s}\end{array}$} & 10 & & & \\
\hline & 100 & A & & \\
\hline
\end{tabular}

Inter-stimulus interval $0.05 \mathrm{~ms}$, amplitude $10^{\wedge} 8 \mathrm{mV} / \mathrm{cm}^{2}$

\begin{tabular}{|c|c|c|c|c|}
\multicolumn{2}{c|}{} & \multicolumn{3}{c|}{ Number of pulses } \\
\cline { 3 - 5 } \multicolumn{1}{c|}{} & 2 & 5 & 10 \\
\hline Pulse & 10 & & & \\
\cline { 4 - 5 } width, $\mu \mathrm{s}$ & 100 & & & \\
\cline { 2 - 5 } & & &
\end{tabular}

Figure 12. The summary of an axonal action potential generation of C-Fibre model for a range of number of pulses, pulse widths and stop pauses. Red colour means no AP was generated, green AP was generated. Frequency $1 \mathrm{MHz}$, stop phase $\pi$. A) $0.01 \mathrm{~ms}$ interstimulus interval and amplitude $10^{6} \frac{\mathrm{mV}}{\mathrm{cm}^{2}}$ (0.4 deformation); B) $0.05 \mathrm{~ms}$ interstimulus interval and amplitude $\left.10^{6} \frac{\mathrm{mV}}{\mathrm{cm}^{2}} ; C\right) 0.01 \mathrm{~ms}$ interstimulus interval and amplitude $10^{7} \frac{\mathrm{mV}}{\mathrm{cm}^{2}}$ (0.67 deformation); D) $0.05 \mathrm{~ms}$ interstimulus interval and amplitude $10^{7} \frac{\mathrm{mV}}{\mathrm{cm}^{2}}$; E) $0.01 \mathrm{~ms}$ interstimulus interval and amplitude 5 . $10^{7} \frac{\mathrm{mV}}{\mathrm{cm}^{2}}$ (0.8 deformation); F) $0.05 \mathrm{~ms}$ interstimulus interval and amplitude $\left.5 \cdot 10^{7} \frac{\mathrm{mV}}{\mathrm{cm}^{2}} ; \mathrm{G}\right) 0.01$ ms interstimulus interval and amplitude $10^{8} \frac{\mathrm{mV}}{\mathrm{cm}^{2}}$ (0.83 deformation); H) $0.05 \mathrm{~ms}$ interstimulus interval and amplitude $10^{8} \frac{\mathrm{mV}}{\mathrm{cm}^{2}}$.

iv. Phase - probability relationship: prediction of the experimental outcomes given random phase distribution per pulse.

In real experiments, the stop phase of the ultrasound sometimes is not controlled precisely and is therefore randomly distributed between the ultrasound pulses. This was modelled in the current subsection (Figure 13) with the ultrasound pulses at a frequency of $1 \mathrm{MHz}$ with the amplitude of $10^{6} \frac{\mathrm{mV}}{\mathrm{cm}^{2}}(0.43$ strain), and with the $0.01 \mathrm{~ms}$ interstimulus interval. The number of pulses and pulse width were varied respectively.

The stimulation of an axon had probabilistic nature, and the probability of AP generation significantly increased with a higher number of pulses (10 and 20$)$ and smaller pulse widths (mostly 10 and 50 periods, Figure 13). 


\section{Random stop phase study 1}

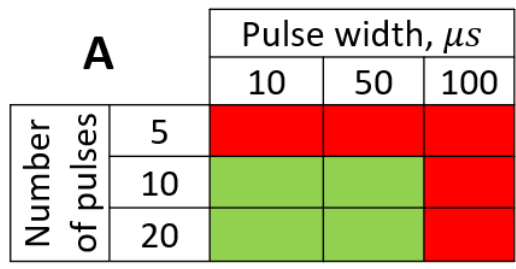

Random stop phase study 2

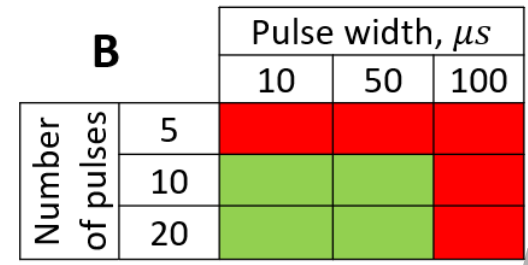

Random stop phase study 3

\begin{tabular}{|c|c|c|c|c|}
\hline \multirow{2}{*}{\multicolumn{2}{|c|}{ C }} & \multicolumn{3}{|c|}{ Pulse width, $\mu s$} \\
\hline & & 10 & 50 & 100 \\
\hline \multirow{3}{*}{ 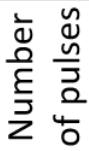 } & 5 & & & \\
\hline & 10 & & & \\
\hline & 20 & & & \\
\hline
\end{tabular}

Random stop phase study 4

\begin{tabular}{|c|c|c|c|c|}
\hline \multirow{2}{*}{\multicolumn{2}{|c|}{ D }} & \multicolumn{3}{|c|}{ Pulse width, $\mu s$} \\
\hline & & 10 & 50 & 100 \\
\hline \multirow{3}{*}{ 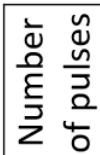 } & 5 & & & \\
\hline & 10 & & & \\
\hline & 20 & & & \\
\hline
\end{tabular}

Random stop phase study 5

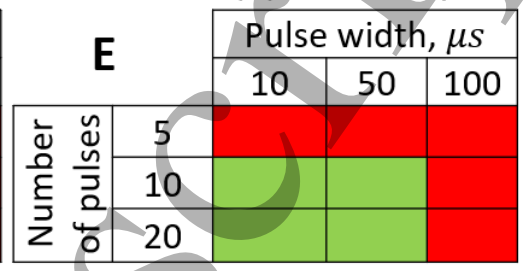

Figure 13. The summary of an axonal action potential generation for a range of the number of pulses and pulse widths of a standing wave pulsed activating function boundary conditions (eq.12) with random stop phases. Red colour means no AP was generated, green - AP was generated. The frequency was $1 \mathrm{MHz}$, amplitude was $10^{6} \frac{\mathrm{mV}}{\mathrm{cm}^{2}}$, interstimulus interval was $0.01 \mathrm{~ms}$. 5 cases with the same ultrasound stimulation parameters except for a random seed number generating random stop phases were simulated. A-E) Studies 1-5.

\section{Discussion}

\section{A) Summary of the results}

The model of ionic concentration changes as a means of AP generation during ultrasound stimulation showed that this mechanism could indeed theoretically be responsible for ultrasound neuromodulation in a variety of cases. The continuous ultrasound case showed that the activating function amplitudes required for AP generation were $10^{5} \frac{\mathrm{mV}}{\mathrm{cm}^{2}}$ and $10^{8} \frac{\mathrm{mV}}{\mathrm{cm}^{2}}$ at $100 \mathrm{kHz}$, and $10^{7} \frac{\mathrm{mV}}{\mathrm{cm}^{2}}$ and $10^{10} \frac{\mathrm{mV}}{\mathrm{cm}^{2}}$ at $1 \mathrm{MHz}$ for $\mathrm{HH}$ and C-fibre models respectively. This corresponded to the strain values of 0.68 and 0.96 at $100 \mathrm{kHz}$ and 0.68 and 0.96 at $1 \mathrm{MHz}$. The strains were equal at both frequencies, that indirectly supported the deformative nature of the effect of ultrasound stimulation on axons.

The pulsed ultrasound stimulation cases showed that the activation threshold varied with the stop phase, the number of pulses in the train, pulse width, and inter-stimulus interval. The stop phase had the most significant effect on the overall probability of AP generation, with the highest probability at the stop phase of $\pi$ for both $\mathrm{HH}$ and C-fibre models.

The lowest thresholds for the AP generation with the pulsed ultrasound were $10^{6} \frac{\mathrm{mV}}{\mathrm{cm}^{2}}$ and 5 . $10^{7} \frac{\mathrm{mV}}{\mathrm{cm}^{2}}$ at $1 \mathrm{MHz}$ for $\mathrm{HH}$ and C-fibre models respectively, corresponded to the strain values of 0.41 and 0.8 , using the 5 pulses with 10 periods each, which were all stopped at $\pi$ stop phase. The analysis of the energy transfer in the system indicated that the first half of the sine wave generated the positive activating function which had a cumulative effect on the generation of the action potential. 
1. Can this mechanism be responsible for AP generation by ultrasound stimulation?

The feasibility and applicability of our results depend strongly on the assumption about the mechanical properties of the neural tissue, in particular the bulk compression modulus $K$. The literature proposes a range of contradicting results, with the values of $\mathrm{K}$ starting from $1 \mathrm{kPa}$ (obtained from the measured elastic modulus and Poisson's ratio under the assumption that the brain tissue is an isotropic linear elastic body) [44], and up to the highest value 2.7 GPa, obtained from the measurement of the speed of ultrasound wave propagation [39].

This in turn leads to a range of possible amplitudes of mechanical strains. On one hand, if $K$ takes a relatively small value, acoustic pressures typically produced by ultrasound stimulating machines could lead to extremely large deformations (strains close to $1(100 \%)$ ). On the other hand, probably the more realistic values of mechanical properties are close to those of water, That is also supported by ultrasound propagation speed [36]-[39], and in this case, typical ultrasound pressures cannot induce strains above $0.01(1 \%)$. According to this assumption, the concentration hypothesis does not produce any significant effects because deformations in the tissue cannot reach high enough values to achieve a certain level of activating function amplitude. However, in such a situation, the effect of cavitation can occur which would drive the deformations high enough to be the prime cause for AP generation, even before the intermembrane cavitation happens.

Other studies have shown that high enough strains are possible in the brain tissue [41]-[45], and thus the ionic concentration effect could as well be responsible for the observed experimental effects. Concerning the peripheral nerve axons specifically, the existing literature suggests that strains can reach 1 (100\%) under the application of the ultrasound [46], [47], which would match our predictions. However, this requires further verification and additional carefully conducted and controlled experiments.

Moreover, the acoustic radiation force, which can be computed as a dependant parameter in our study was shown to be the prime factor causing neurostimulation according to [56]. This radiation force may cause deformations in an axon and its surroundings which may lead to concentration changes. This is supported by the fact that in [56] neurostimulation effect increases with an increase in the ultrasound frequency, which was also observed in the present study.

Further experiments are required to confirm or reject the possibility of the concentration mechanism to be responsible for AP generation by ultrasound stimulation.

2. What are the implications for the experimental predictions?

Although this study could not provide an exact answer to this question due to the absence of the direct experimental evidence, our results could explain and simulate a range of observed experimental effects (see below) without involving any alternative mechanisms, and with specific tuning could also seamlessly incorporate effects of the cavitation. The qualitative behaviour of the AP generation predicted by the approach matched closely the range of experimentally observed effects. Specifically, the increase of neurostimulation effect with the increase of the frequency [56], strong dependence of the effect of ultrasound on its intensity, the increase of neurostimulation effect with the decrease of the pulse duration [5], [47], [59] and the possibility to initiate the AP with low duration pulses [2], [57]. In addition, the probabilistic nature of ultrasound stimulation of unmyelinated peripherical nerves shown in [59] was directly predicted by our model when random stop phase was employed, that simulated the realistic ultrasound parameters. The small qualitative difference there could be explained by different axon parameters. 
Our approach is based on a conventional electrical stimulation model, which fits well with the experimental evidence showing that ultrasound and electrical stimulation methods have common effects on axons [47]. Finally, experiments show [58] that membrane potential depends on strain and strain rate which corresponds well to the proposed mechanism and also validates the dependence of AP generation on a frequency of ultrasound.

The results and the models can be used directly to create a realistic FE model of ultrasound stimulation of a subdiaphragmatic vagus nerve and nociceptors. It is planned to directly verify our results in subdiaphragmatic vagus nerve through ex vivo and in vivo experiments.

Specific attention should be brought to the obtained activating-phase relationship, which favours phases close to $\pi$. This, together with the probabilistic nature of pulsed ultrasound phase control, could explain the apparent probabilistic nature of the AP generation in the experiments mentioned by [3], [54], [57].

We are planning to design an experiment, where the phase of the ultrasound will be controlled precisely. This could decisively test the question about the prime mechanisms responsible for neurostimulation because if this is intermembrane cavitation, the results would stay probabilistic even in the controlled case conditions, contrary to the proposed approach where results would strongly depend on the phase.

We are also planning to design the experiment for the exact measurement of the strain occurring in neural tissue during the propagation of the ultrasound which could point to the feasibility and applicability of the proposed approach.

\section{C) Technical limitations.}

Here we only considered unmyelinated fibres. We also greatly simplified the transition between mechanical strain and boundary conditions. Although we are confident about the qualitative results, we need to develop a more complicated model in order to study the quantitative effects thoroughly. The one-to-one comparison to other hypotheses would be ideal, however, this is challenging given the fact that most parameters for the other hypotheses are usually obtained experimentally.

\section{D) Future work.}

In future, we are planning to extend the model for the simultaneous solution of Nernst-Plank equations and $\mathrm{HH}$ (and C-fibre) equations. We are also planning to create a myelinated coupled electro-acoustic model of the axon. We then are planning to confirm the qualitative results obtained in this work, obtain predictive quantitative results for particular nerves, and conduct an experimental study which would definitively answer the question on the exact influence of the ionic concentration mechanism on neurostimulation by the ultrasound.

One of the potential applications of ultrasound stimulation could be the possibility of affecting the direction of the information flow within the vagus nerve. For example, the threshold of stimulation could be different for A-delta efferent fibres and the rest of the myelinated fibres. Existing models of myelinated fibres [60] offer the possibility of such investigations. We therefore will consider the possibility of assessing this and comparing the myelinated and unmyelinated fibre behaviour.

5. Acknowledgement 
This research work was supported by the Academic Excellence Project 5-100 proposed by Peter the Great St. Petersburg Polytechnic University.

6. Literature

[1] M. L. Khraiche, W. B. Phillips, N. Jackson, and J. Muthuswamy, "Ultrasound induced increase in excitability of single neurons," in Proceedings of the 30th Annual International Conference of the IEEE Engineering in Medicine and Biology Society, EMBS'08 - "Personalized Healthcare through Technology," 2008, vol. 2008, pp. 4246-4249, doi: 10.1109/iembs.2008.4650147.

[2] W. J. Tyler, Y. Tufail, M. Finsterwald, M. L. Tauchmann, E. J. Olson, and C. Majestic, "Remote Excitation of Neuronal Circuits Using Low-Intensity, Low-Frequency Ultrasound," PLoS One, vol. 3, no. 10, p. e3511, Oct. 2008, doi: 10.1371/journal.pone.0003511.

[3] J. Blackmore, S. Shrivastava, J. Sallet, C. R. Butler, and R. O. Cleveland, "Ultrasound Neuromodulation: A Review of Results, Mechanisms and Safety," Ultrasound in Medicine and Biology, vol. 45, no. 7. Elsevier USA, pp. 1509-1536, Jul. 01, 2019, doi: 10.1016/j.ultrasmedbio.2018.12.015.

[4] B. Feng, L. Chen, and S. J. Ilham, "A review on ultrasonic neuromodulation of the peripheral nervous system: Enhanced or suppressed activities?," Applied Sciences (Switzerland), vol. 9, no. 8. MDPI AG, Apr. 01, 2019, doi: 10.3390/app9081637.

[5] C. J. Wright, J. Rothwell, and N. Saffari, "Ultrasonic stimulation of peripheral nervous tissue: An investigation into mechanisms," in Journal of Physics: Conference Series, Jan. 2015, vol. 581, no. 1, p. 012003, doi: 10.1088/1742-6596/581/1/012003.

[6] E. Kimmel, "Cavitation bioeffects," Critical Reviews in Biomedical Engineering, vol. 34, no. 2. Begell House Inc., pp. 105-161, 2006, doi: 10.1615/critrevbiomedeng.v34.i2.10.

[7] J. Gateau, J. F. Aubry, D. Chauvet, A. L. Boch, M. Fink, and M. Tanter, "In vivo bubble nucleation probability in sheep brain tissue," Phys. Med. Biol., vol. 56, no. 22, pp. 7001-7015, Nov. 2011, doi: 10.1088/0031-9155/56/22/001.

[8] N. I. Vykhodtseva, K. Hynynen, and C. Damianou, "Histologic effects of high intensity pulsed ultrasound exposure with subharmonic emission in rabbit brain in vivo," Ultrasound Med. Biol., vol. 21, no. 7, pp. 969-979, Jan. 1995, doi: 10.1016/0301-5629(95)00038-S.

[9] B. Martinac, "Mechanosensitive ion channels: Molecules of mechanotransduction," J. Cell Sci., vol. 117, no. 12, pp. 2449-2460, May 2004, doi: 10.1242/jcs.01232.

[10] F. Sachs, "Stretch-activated ion channels: What are they?," Physiology, vol. 25, no. 1. Physiology (Bethesda), pp. 50-56, Feb. 2010, doi: 10.1152/physiol.00042.2009.

[11] M. Plaksin, S. Shoham, and E. Kimmel, "Intramembrane cavitation as a predictive biopiezoelectric mechanism for ultrasonic brain stimulation," Phys. Rev. $X$, vol. 4, no. 1, p. 011004, Jan. 2014, doi: 10.1103/PhysRevX.4.011004.

[12] B. Krasovitski, V. Frenkel, S. Shoham, and E. Kimmel, "Intramembrane cavitation as a unifying mechanism for ultrasound-induced bioeffects," Proc. Natl. Acad. Sci. U. S. A., vol. 108, no. 8 , pp. 3258-3263, Feb. 2011, doi: 10.1073/pnas.1015771108.

[13] T. Tarnaud, W. Joseph, R. Schoeters, L. Martens, and E. Tanghe, "SECONIC: Towards multicompartmental models for ultrasonic brain stimulation by intramembrane cavitation," J. Neural Eng., vol. 17, no. 5, p. 056010, Oct. 2020, doi: 10.1088/1741-2552/abb73d. 
[14] A. Delalande, S. Kotopoulis, T. Rovers, C. Pichon, and M. Postema, "Sonoporation at a low mechanical index," Bubble Sci. Eng. Technol., vol. 3, no. 1, pp. 3-11, May 2011, doi: 10.1179/1758897911Y.0000000001.

[15] E. Sassaroli and N. Vykhodtseva, "Acoustic neuromodulation from a basic science prospective," Journal of Therapeutic Ultrasound, vol. 4, no. 1. BioMed Central Ltd., 2016, doi: 10.1186/s40349-016-0061-z.

[16] H. A. S. Kamimura, A. Conti, N. Toschi, and E. E. Konofagou, "Ultrasound Neuromodulation: Mechanisms and the Potential of Multimodal Stimulation for Neuronal Function Assessment," Front. Phys., vol. 8, p. 150, May 2020, doi: 10.3389/FPHY.2020.00150.

[17] B. Wunderlich et al., "Phase-state dependent current fluctuations in pure lipid membranes," Biophys. J., vol. 96, no. 11, pp. 4592-4597, Jun. 2009, doi: 10.1016/j.bpj.2009.02.053.

[18] V. F. Antonov, E. V. Shevchenko, E. T. Kozhomkulov, A. A. Mol'nar, and E. Y. Smirnova, "Capacitive and ionic currents in BLM from phosphatidic acid in Ca2+-induced phase transition," Biochem. Biophys. Res. Commun., vol. 133, no. 3, pp. 1098-1103, Dec. 1985, doi: 10.1016/0006-291X(85)91249-5.

[19] R. A. Chapman, "Dependence on temperature of the conduction velocity of the action potential of the squid giant axon [39]," Nature, vol. 213, no. 5081. Nature Publishing Group, pp. 1143-1144, Mar. 01, 1967, doi: 10.1038/2131143a0.

[20] M. Chernov and A. W. Roe, "Infrared neural stimulation: a new stimulation tool for central nervous system applications," Neurophotonics, vol. 1, no. 1, p. 011011, Aug. 2014, doi: 10.1117/1.nph.1.1.011011.

[21] I. Lenart and D. Ausländer, "The effect of ultrasound on diffusion through membranes," Ultrasonics, vol. 18, no. 5, Sep. 1980, doi: 10.1016/0041-624X(80)90123-7.

[22] D. Ma, J. S. Marshall, and J. Wu, "Measurement of ultrasound-enhanced diffusion coefficient of nanoparticles in an agarose hydrogel," J. Acoust. Soc. Am., vol. 144, no. 6, pp. 3496-3502, Dec. 2018, doi: 10.1121/1.5083828.

[23] N. Thompson, S. Mastitskaya, and D. Holder, "Avoiding off-target effects in electrical stimulation of the cervical vagus nerve: Neuroanatomical tracing techniques to study fascicular anatomy of the vagus nerve," Journal of Neuroscience Methods, vol. 325. Elsevier B.V., Sep. 01, 2019, doi:10.1016/j.jneumeth.2019.108325.

[24] L. N. Long and G. Fang, "A review of biologically plausible neuron models for spiking neural networks," AIAA Infotech Aerosp. 2010, 2010, doi: 10.2514/6.2010-3540.

[25] S. Terakawa and A. Watanabe, "Electrical responses to mechanical stimulation of the membrane of squid giant axons," Pflügers Arch. Eur. J. Physiol., vol. 395, no. 1, pp. 59-64, Oct. 1982, doi: 10.1007/BF00584969.

[26] W. Legon, A. Rowlands, A. Opitz, T. F. Sato, and W. J. Tyler, "Pulsed Ultrasound Differentially Stimulates Somatosensory Circuits in Humans as Indicated by EEG and fMRI," PLoS One, vol. 7, no. 12, p. e51177, Dec. 2012, doi: 10.1371/journal.pone.0051177.

[27] E. J. Juan, R. González, G. Albors, M. P. Ward, and P. Irazoqui, "Vagus nerve modulation using focused pulsed ultrasound: Potential applications and preliminary observations in a rat," Int. J. Imaging Syst. Technol., vol. 24, no. 1, pp. 67-71, Mar. 2014, doi: 10.1002/ima.22080.

[28] K. M. Wasilczuk, "Low Intensity Focused Ultrasound Stimulation of the Vagus Nerve for Modulating the Inflammatory Reflex Assessed in Rat Model," Theses Diss. Available from 
ProQuest, Jan. 2018, Accessed: Jul. 14, 2021. [Online]. Available: https://docs.lib.purdue.edu/dissertations/AAl10808112.

[29] N. Ji, W.-H. Lin, F. Chen, L. Xu, J. Huang, and G. Li, "Blood Pressure Modulation With LowIntensity Focused Ultrasound Stimulation to the Vagus Nerve: A Pilot Animal Study," Front. Neurosci., vol. 14, p. 1125, Nov. 2020, doi: 10.3389/FNINS.2020.586424.

[30] C. CR, K. A, X. W, B. M, A. ST, and R. AJ, "Chronic Vagus Nerve Stimulation Significantly Improves Quality of Life in Treatment-Resistant Major Depression," J. Clin. Psychiatry, vol. 79, no. 5, Sep. 2018, doi: 10.4088/JCP.18M12178.

[31] A. L. Hodgkin and A. F. Huxley, "A quantitative description of membrane current and its application to conduction and excitation in nerve," J. Physiol., vol. 117, no. 4, pp. 500-544, Aug. 1952, doi: 10.1113/jphysiol.1952.sp004764.

[32] J. Tigerholm et al., "Modeling activity-dependent changes of axonal spike conduction in primary afferent C-nociceptors," J. Neurophysiol., vol. 111, no. 9, pp. 1721-1735, May 2014, doi: 10.1152/jn.00777.2012.

[33] J. Pods, J. Schönke, and P. Bastian, "Electrodiffusion models of neurons and extracellular space using the poisson-nernst-planck equations - Numerical simulation of the intra- and extracellular potential for an axon model," Biophys. J., vol. 105, no. 1, pp. 242-254, Jul. 2013, doi: 10.1016/j.bpj.2013.05.041.

[34] Q. Zheng and G. W. Wei, "Poisson-Boltzmann-Nernst-Planck model," J. Chem. Phys., vol. 134, no. 19 , May 2011, doi: 10.1063/1.3581031.

[35] M. S. Canney, M. R. Bailey, L. A. Crum, V. A. Khokhlova, and O. A. Sapozhnikov, "Acoustic characterization of high intensity focused ultrasound fields: A combined measurement and modeling approach," J. Acoust. Soc. Am., vol. 124, no. 4, pp. 2406-2420, Oct. 2008, doi: 10.1121/1.2967836.

[36] S. Ganpule, N. P. Daphalapurkar, M. P. Cetingul, and K. T. Ramesh, "Effect of bulk modulus on deformation of the brain under rotational accelerations.," Shock waves, vol. 28, no. 1, pp. 127-139, Jan. 2018, doi: 10.1007/s00193-017-0791-z.

[37] I. Zoric, B. Ivancevic, and K. Jambrosic, "The velocity of ultrasound propagation through brain tissue at low ultrasound frequencies," J. Acoust. Soc. Am., vol. 105, no. 2, pp. 1370-1370, Feb. 1999, doi: 10.1121/1.426486.

[38] C. J. Reyes Hernandez, L. Leija Salas, and A. Vera Hernandez, "Measurement of ultrasound speed and attenuation coefficient of brain phantom using pulse echo and through transmission method," in 2018 Global Medical Engineering Physics Exchanges/Pan American Health Care Exchanges, GMEPE/PAHCE 2018, Jun. 2018, pp. 1-4, doi: 10.1109/GMEPEPAHCE.2018.8400764.

[39] A. Etoh, S. Mitaku, J. Yamamoto, and K. Okano, "Ultrasonic absorption anomaly of brain tissue," Jpn. J. Appl. Phys., vol. 33, no. 5S, pp. 2874-2879, May 1994, doi: 10.1143/JJAP.33.2874.

[40] L. R. Gavrilov, G. V Gersuni, O. B. Ilyinski, E. M. Tsirulnikov, and E. E. Shchekanov, "A study of reception with the use of focused ultrasound. I. Effects on the skin and deep receptor structures in man," 1977.

[41] F. Morin, M. Chabanas, H. Courtecuisse, and Y. Payan, "Biomechanical Modeling of Brain Soft Tissues for Medical Applications," in Biomechanics of Living Organs: Hyperelastic Constitutive Laws for Finite Element Modeling, Elsevier Inc., 2017, pp. 127-146. 
[42] G. Soza, R. Grosso, C. Nimsky, G. Greiner, and P. Hastreiter, "Estimating mechanical brain tissue properties with simulation and registration," in Lecture Notes in Computer Science, 2004, vol. 3217, no. 1 PART 2, pp. 276-283, doi: 10.1007/978-3-540-30136-3_35.

[43] K. Miller, K. Chinzei, G. Orssengo, and P. Bednarz, "Mechanical properties of brain tissue invivo: Experiment and computer simulation," J. Biomech., vol. 33, no. 11, pp. 1369-1376, Nov 2000, doi: 10.1016/S0021-9290(00)00120-2.

[44] K. Miller and K. Chinzei, "Mechanical properties of brain tissue in tension," J. Biomech., vol. 35, no. 4, pp. 483-490, Apr. 2002, doi: 10.1016/\$0021-9290(01)00234-2.

[45] K. Laksari, M. Shafieian, and K. Darvish, "Constitutive model for brain tissue under finite compression," J. Biomech., vol. 45, no. 4, pp. 642-646, Feb. 2012, doi: 10.1016/j.jbiomech.2011.12.023.

[46] F. J. Julian and D. E. Goldman, "The effects of mechanical stimulation on some electrical properties of axons," J. Gen. Physiol., vol. 46, no. 2, pp. 297-313, Nov. 1962, doi: 10.1085/jgp.46.2.297.

[47] M. E. Downs, S. A. Lee, G. Yang, S. Kim, Q. Wang, and E. E. Konofagou, "Non-invasive peripheral nerve stimulation via focused ultrasound in vivo," Phys. Med. Biol., vol. 63, no. 3, p. 35011, Feb. 2018, doi: 10.1088/1361-6560/aa9fc2.

[48] Bertil Hille, Ion Channels of Excitable Membranes, Third Edition. 2001.

[49] F. Rattay, "Analysis of Models for Extracellular Fiber Stimulation," IEEE Trans. Biomed. Eng., vol. 36, no. 7, pp. 676-682, 1989, doi: 10.1109/10.32099.

[50] A. L. HODGKIN and W. A. RUSHTON, "The electrical constants of a crustacean nerve fibre," Proc. R. Soc. London. Ser. B - Biol. Sci., vol. 133, no. 873, pp. 444-479, Dec. 1946, doi: 10.1098/rspb.1946.0024.

[51] W. Rall, "Branching dendritic trees and motoneuron membrane resistivity," Exp. Neurol., vol. 1, no. 5, pp. 491-527, Nov. 1959, doi: 10.1016/0014-4886(59)90046-9.

[52] I. Tarotin, K. Aristovich, and D. Holder, "Model of impedance changes in unmyelinated nerve fibers," IEEE Trans. Biomed. Eng., vol. 66, no. 2, pp. 471-484, Feb. 2019, doi: 10.1109/TBME.2018.2849220.

[53] H. Kim, A. Chiu, S. D. Lee, K. Fischer, and S.-S. Yoo, "Focused Ultrasound-mediated Noninvasive Brain Stimulation: Examination of Sonication Parameters," Brain Stimul., vol. 7, no. 5, Sep. 2014, doi: 10.1016/j.brs.2014.06.011.

[54] R. L. King, J. R. Brown, W. T. Newsome, and K. B. Pauly, "Effective Parameters for UltrasoundInduced In Vivo Neurostimulation," Ultrasound Med. Biol., vol. 39, no. 2, Feb. 2013, doi: 10.1016/j.ultrasmedbio.2012.09.009.

[55] J. J. C. Rosenthal and F. Bezanilla, "Seasonal variation in conduction velocity of action potentials in squid giant axons," Biol. Bull., vol. 199, no. 2, pp. 135-143, 2000, doi: $10.2307 / 1542873$.

[56] M. D. Menz et al., "Radiation Force as a Physical Mechanism for Ultrasonic Neurostimulation of the Ex Vivo Retina," J. Neurosci., vol. 39, no. 32, Aug. 2019, doi: 10.1523/JNEUROSCI.239418.2019.

[57] J. Vion-Bailly, W. A. N'Djin, I. M. Suarez Castellanos, J.-L. Mestas, A. Carpentier, and J.-Y. Chapelon, "A causal study of the phenomenon of ultrasound neurostimulation applied to an in vivo invertebrate nervous model," Sci. Rep., vol. 9, no. 1, Dec. 2019, doi: 10.1038/s41598- 
019-50147-7.

[58] J. A. Galbraith, L. E. Thibault, and D. R. Matteson, "Mechanical and electrical responses of the squid giant axon to simple elongation," J. Biomech. Eng., vol. 115, no. 1, pp. 13-22, Feb. 1993, doi: 10.1115/1.2895464.

[59] C. J. Wright, S. R. Haqshenas, J. Rothwell, and N. Saffari, "Unmyelinated Peripheral Nerves Can Be Stimulated in Vitro Using Pulsed Ultrasound," Ultrasound Med. Biol., vol. 43, no. 10, pp. 2269-2283, Oct. 2017, doi: 10.1016/J.ULTRASMEDBIO.2017.05.008.

[60] C. C. McIntyre, A. G. Richardson, and W. M. Grill, "Modeling the excitability of mammalian nerve fibers: influence of afterpotentials on the recovery cycle.," J. Neurophysiol., vol. 87, no. 2, pp. 995-1006, 2002, doi: 10.1152/jn.00353.2001. 